\title{
Influence of the interface corrugation on the subband dispersions and the optical properties of (113)-oriented GaAs/AlAs superlattices
}

\author{
Langbein, Wolfgang Werner; Lüerssen, D.; Kalt, H.; Hvam, Jørn Märcher; Braun, W.; Ploog, K.
}

Published in:

Physical Review B

Link to article, DOI:

10.1103/PhysRevB.54.10784

Publication date:

1996

Document Version

Publisher's PDF, also known as Version of record

Link back to DTU Orbit

Citation (APA):

Langbein, W. W., Lüerssen, D., Kalt, H., Hvam, J. M., Braun, W., \& Ploog, K. (1996). Influence of the interface corrugation on the subband dispersions and the optical properties of (113)-oriented GaAs/AlAs superlattices. Physical Review B, 54(15), 10784-10799. https://doi.org/10.1103/PhysRevB.54.10784

\section{General rights}

Copyright and moral rights for the publications made accessible in the public portal are retained by the authors and/or other copyright owners and it is a condition of accessing publications that users recognise and abide by the legal requirements associated with these rights.

- Users may download and print one copy of any publication from the public portal for the purpose of private study or research.

- You may not further distribute the material or use it for any profit-making activity or commercial gain

- You may freely distribute the URL identifying the publication in the public portal 


\title{
Influence of the interface corrugation on the subband dispersions and the optical properties of (113)-oriented GaAs/AlAs superlattices
}

\author{
W. Langbein,* D. Lüerßen, and H. Kalt \\ Institut für Angewandte Physik, Universität Karlsruhe, D-76128 Karlsruhe, Germany \\ J. M. Hvam \\ Mikroelektronik Centret, The Technical University of Denmark, Bldg. 345 east, DK-2800 Lyngby, Denmark \\ W. Braun and K. Ploog \\ Paul-Drude-Institut für Festkörperelektronik, D-10117 Berlin, Germany
}

(Received 27 March 1996)

\begin{abstract}
We report on the influence of the interface corrugation in (113)-grown GaAs/AlAs superlattices on their band-edge optical properties both in theory and experiment. We calculate the subband dispersions and the optical anisotropies in a multiband $\mathbf{k} \cdot \mathbf{p}$ formalism. The dominating contribution to the optical anisotropies is found to be due to the intrinsic properties of the valence-band structure. The corrugation modifies the density of states only slightly, giving no evidence of a quantum-wire behavior. By comparing the calculation with the experimental optical anisotropy, we can estimate the corrugation height to be at most 2 monolayers. The experiments show that deviations from the regular corrugation lead to an anisotropic interface disorder. This gives rise to an enhanced anisotropy of the band-edge states, which was so far attributed to the corrugation itself. The luminescence of the localized type-I states at the band-edge show an enhanced optical anisotropy in comparison to the luminescence of the extended states, revealing the anisotropic nature of their localization sites. In type-II samples, deeply localized, isolated type-I states ( $\Gamma$ quantum boxes) dominate the luminescence at short delays after pulsed excitation and at higher lattice temperatures or excitation densities, due to their strong radiative decay compared to the type-II states. These quantum boxes are observed individually by high spatial and spectral resolution. [S0163-1829(96)06039-0]
\end{abstract}

\section{INTRODUCTION}

A current topic of interest in semiconductor physics is the investigation of semiconductor nanostructures, exhibiting a reduction of the effective dimensionality of the dispersion of the electronic states. This reduction results in a concentration of the density of states at the band edge and in reduced scattering in the remaining directions of free motion, which is both of interest for optical and electrical devices such as laser diodes and fast transistors. ${ }^{1-4}$ It can be achieved by structural sizes in the nanometer range, resulting in an energy difference of the confined states larger than both the thermal energy and the excitonic binding energy.

The fabrication of quasi-two-dimensional structures can be achieved by the growth of thin films with near-monolayer thickness control. The further reduction in dimensionality to quantum wire structures is more complicated. The fabrication by lithographic methods is limited to an accuracy of about ten nanometers, and thus gives rise to a strong inhomogeneous broadening of the electronic properties. ${ }^{5-7}$ There are some methods to overcome this difficulty, e.g., by the overgrowth of cleaved quantum-well structures ${ }^{8-11}$ as well as etched $V$ grooves on GaAs substrates. ${ }^{2,12}$ However, these methods require involved processing.

A more direct way to obtain one-dimensional structures would be the use of the self-organized facetting of highindex surfaces, as proposed by Nötzel et al. ${ }^{13}$ The (113) surface of GaAs was reported to show a spontaneous corruga- tion along the $[1 \overline{1} 0]$ direction with a period of $3.2 \mathrm{~nm}$ and a height of $1.02 \mathrm{~nm},{ }^{14-16}$ leading to the formation of quantumwell wires during thin-film growth by molecular beam epitaxy (MBE). The instability of the flat (113) surface against this corrugation was attributed to a difference in the surface free energy. ${ }^{17}$

The actual height of the corrugation and even its presence were controversially discussed in the recent literature. The corrugated surface reconstruction is not observed in atomic layer (AL)-MBE (Ref. 18) and under arsenic-rich conditions. ${ }^{19}$ Raman experiments by Armelles et al. ${ }^{20}$ on AL-MBE and MBE-grown samples only revealed a higher degree of interface disorder in the MBE samples. On the other hand, the observation of confined optical ${ }^{21}$ and acoustical $^{22}$ phonons experimentally verified the presence of some interface corrugation with a period of $3.2 \mathrm{~nm}$ at the superlattice heterointerfaces in samples grown by Nötzel and Ploog.

The corrugated structures show an enhanced exciton binding energy and hot exciton luminescence, which was attributed to the one-dimensional density of states. ${ }^{23}$ However, these results can also be explained in terms of a strong exciton localization ${ }^{24}$ in these superlattices (SL's). The corrugation height of 6 monolayers (ML) or $1.02 \mathrm{~nm}$ was estimated from in situ reflection high-energy electron diffraction (RHEED) on the surface. Results obtained by scanning tunneling microscopy (STM) (Ref. 25) suggest a significantly smaller corrugation height of about 2 ML. This surface re- 
construction, which is consistent with the electron counting model, ${ }^{25,26}$ can lead to the reported ${ }^{13}$ RHEED signal, as was shown by a kinematical simulation. ${ }^{27}$ Recently, da Silva et al. also estimated the interface corrugation height to be $2 \mathrm{ML}$ by the splitting of the confined optical phonons. ${ }^{28}$

A significant body of recent research work is devoted to observe optical anisotropies in the photoluminescence (PL), the PL excitation (PLE), ${ }^{23,29}$ reflectance, ${ }^{30,31}$ photoreflectance, ${ }^{32}$ and the piezoreflectance ${ }^{20}$ of the (113)-grown SL's. Such anisotropies are due to the valence-band structure of GaAs, which leads to a dependence of the SL in-plane dispersion $^{33}$ and in-plane optical anisotropy ${ }^{34,35}$ on the growth direction.

In this paper, we review our studies of the optical anisotropy in the (113)-grown GaAs/AlAs SL system. We investigate a series of SL's with different layer thicknesses, grown by molecular-beam epitaxy (MBE), showing the $(8 \times 1)$ reconstruction ${ }^{13,25}$ in in situ RHEED. We compare the polarization degree (PD) of the PL with the results of multiband $\mathbf{k} \cdot \mathbf{p}$ calculations of the electronic states and optical transitions near the fundamental band gap. The corrugation present in the samples is found to have only a minor influence on the optical anisotropy of transitions between extended states. Deviations from the strict periodicity of the corrugation lead to the formation of anisotropically localized electronic states, exhibiting an enhanced optical anisotropy.

The paper is organized as follows: In Sec. II we describe the theoretical model for the calculation of the quantized electronic states both in the conduction and valence bands as well as the optical anisotropy of the interband transitions. We show and discuss the numerical results for different sample parameters. In Sec. III we compare the experimental PD of the absorption, PLE, and PL spectra with the calculated PD, which allows us to estimate the corrugation height present in the samples to be $2 \mathrm{ML}$ at most. In Sec. IV we show the existence of anisotropically localized band-edge states and investigate their influence on the PL dynamics in type-I and -II samples. In Sec. V we finally summarize the results.

\section{ELECTRONIC STATES AND OPTICAL TRANSITIONS}

The electronic states in (113)-grown GaAs/AlAs SL's with atomically flat interfaces were theoretically investigated previously both by multiband $\mathbf{k} \cdot \mathbf{p}$ calculations, ${ }^{36}$ tightbinding methods, ${ }^{37,38}$ and pseudopotential calculations. ${ }^{39}$ The hole dispersions calculated in the six-band $\mathbf{k} \cdot \mathbf{p}$ model have been found to be in reasonable agreement with those determined by resonant tunneling experiments. ${ }^{40,41}$ The optical anisotropy of the transitions from the highest valence subbands to the lowest conduction subband, determined by reflection experiments, have been found to be in agreement or somewhat larger than predicted from the calculations employing flat interfaces. ${ }^{20,30,32,42}$

The question of how optical anisotropies in the SL's are changed by the 6-ML antiphase interface corrugation (APC) proposed by Nötzel et al., ${ }^{13}$ and a comparison with the experimental results, has not been addressed in depth until now, to our knowledge. Previous results published for the corrugated SL's can be summarized as follows.

The energies of the zone-center conduction-band states

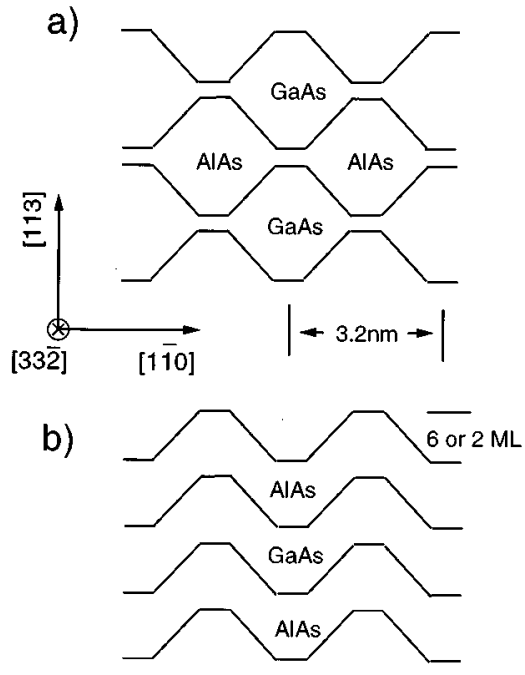

FIG. 1. Schematic representation of the directions and the considered corrugations of the SL structure. (a) Antiphase corrugation (b) In-phase corrugation.

under the presence of 6-ML APC were calculated by Jouanin and Pertho, ${ }^{43}$ who found a small change of the type-I to type-II transition period length. The conduction-band states were treated by Kiselev and Rössler using an effective-mass approach $^{44}$. They found a blueshift of the first subband, and a slightly anisotropic in-plane dispersion of about $10 \%$, leading to a minor increase of the excitonic binding energy. This is in agreement with the results of Langbein, ${ }^{45}$ showing that the corrugation-induced potential is not able to confine the carriers within a period of $3.2 \mathrm{~nm}$, which would involve a kinetic energy of about $100 \mathrm{meV}$ for the heavy hole and 400 $\mathrm{meV}$ for the electron. The valence-band states and the dispersions in SL's with 6-ML APC were calculated by Li and $\mathrm{Xia}^{46}$ using a four-band $\mathbf{k} \cdot \mathbf{p}$ model. Their calculated optical anisotropies were only approximate due to the neglection of the split-off hole. ${ }^{35}$ The calculated excitonic binding energy in the corrugated SL's (Ref. 47) was found to be enhanced by up to $100 \%$. This finding was claimed to be the reason for the redshift of the PL of the (113)-grown SL's compared to the (001)-grown ones. The optical anisotropy measured by reflectance difference spectroscopy was a factor of 3 lower than calculated for 6-ML APC using a tight-binding calculation, ${ }^{31}$ giving evidence for a smaller corrugation height present at the interfaces.

\section{A. Theoretical model}

In this paper, both the interface corrugation and the valence-band structure including the split-off band are taken into account, which is necessary for a good description of the influence of the corrugation on the optical anisotropy of the SL's. Excitonic effects ${ }^{48,49}$ are neglected, because they do not influence the optical anisotropy of the subband transitions in this case. This is due to the small exciton binding energy ${ }^{23,47,50-52}$ in comparison with the subband separations.

The schematic structure of the considered interface corrugation and directions are given in Fig. 1. In the calculations, 
the corrugation height was varied between 0 and $6 \mathrm{ML}$, and the relative phase of the corrugation at the GaAs/AlAs and $\mathrm{AlAs} / \mathrm{GaAs}$ interfaces was chosen to be either antiphase [Fig. 1a] or in-phase [Fig. 1(b)].

To calculate the electronic subbands, we start with the determination of the subband wave functions $\Phi^{n}$ at the zone center $(\Gamma)$ in an effective-mass approach. We discretize one period of the structure in real space to $N_{\mathbf{x}} \times N_{\mathbf{y}}$ points in the directions $\mathbf{x} \equiv[113]$ and $\mathbf{y} \equiv[1 \overline{10}]$, where the numbers $N_{\mathbf{x}, \mathbf{y}}$ are powers of 2 .

In the following, we describe the calculation for onedimensional superlattices in the $\mathrm{z}$ direction, for simplicity. The expansion to the two-dimensional case is straightforward.

As an ansatz for the $\Phi^{n}$, we use the Fourier components $(\cos , \sin )\left[\left(2 \pi n_{\mathrm{z}} / L_{\mathrm{z}}\right) z\right]$ with $n_{z}=0,1, \ldots N_{z} / 2$, excluding the sine components of the lowest and highest momenta, which vanish on the grid in real space. This leads to the eigenvalue equation

$$
\begin{aligned}
& \left(\partial_{z} \frac{\hbar^{2}}{2 m_{\mathrm{z}}(z)} \partial_{z}+V(z)-E_{n}\right) \\
& \quad \times(\underbrace{\left(\sum_{n_{z}=0}^{N_{z} / 2} c_{n_{z}}^{n} \cos \left(\frac{2 \pi n_{z}}{L_{z}} z\right)+s_{n_{z} \neq\left\{0, N_{z} / 2\right\}}^{n} \sin \left(\frac{2 \pi n_{z}}{L_{z}} z\right)\right)}_{\Phi^{n}(z)}=0,
\end{aligned}
$$

with the superlattice period $L_{z}$, the effective mass $m_{z}(z)$, and the eigenenergies $E_{n}$ for the eigenvectors $\left(c^{n}, s^{n}\right)$.

To determine the conduction subband dispersion around the $\Gamma$ point, we use the $M_{\mathrm{z}}=10 \ldots 100$ energetically lowest eigenstates $\Phi^{n}(z)$ of Eq. (1) as ansatz states for the eigenstates $\Psi^{n}(\vec{k}, z)$ with the quasimomentum $\hbar \vec{k} \neq 0$ in the conduction-band Hamiltonian,

$$
\begin{gathered}
\left(\partial_{z} \frac{\hbar^{2}}{2 m_{z}(z)} \partial_{z}+V^{e}(z)-E_{n}^{e}(\vec{k})\right) \\
\times \underbrace{\left[e^{i \vec{k} \cdot \vec{x}}\left(\sum_{m=1}^{M_{z}} a_{m}^{n, \vec{k}} \Phi^{m}(z)\right)\right]}_{\Psi^{n}(\vec{k}, z)}=0
\end{gathered}
$$

The eigenvectors $a^{n, \vec{k}}$ define the eigenstates $\Psi^{n}(\vec{k}, z)$, which are the envelope functions of the $n$th conduction subband with the quasimomentum $\hbar \vec{k}$.

To determine the valence subband states, we start with Eq. (1), using the mean effective mass $1 / m_{\mathrm{z}}(z)=\gamma_{1}(z)$ of the valence bands and the potential $V(z)$ of the heavy-hole band. We use the resulting eigenstates $\Phi^{n}(z)$ as ansatz states for the periodic part of the envelope functions of the six spinorbit-coupled components $|\mu\rangle$ of the valence band, ${ }^{53,54}$

$$
\begin{aligned}
\sum_{\mu^{\prime}=1}^{6} & {\left.\left[T_{\mu, \mu^{\prime}}(z)+\left(V_{\mu}^{h}(z)-E_{n}^{h}\right) \delta_{\mu, \mu^{\prime}}\right)\right] } \\
\times & \underbrace{\left[e^{i \vec{k} \cdot \vec{x}}\left(\sum_{m=1}^{M_{z}} g_{m}^{n, \mu^{\prime}, \vec{k}} \Phi^{m}(z)\right)\right]}_{\Psi_{\mu^{\prime}}^{n}(\vec{k}, z)}=0, \quad \forall_{\mu}
\end{aligned}
$$

The eigenvectors $g^{n, \vec{k}}$ then describe the valence subband wave functions in the $\Phi(z)$ basis. The kinetic operators $T_{\mu, \mu^{\prime}}(z)$ and the potential $V_{\mu}^{h}(z)$ are given by the Luttinger Hamiltonian $\mathcal{H}_{\text {lutt }}$ (Ref. 54):

$$
\mathcal{H}_{\text {lutt }}=E_{\Gamma_{8}}(z)-\left(\begin{array}{cccccc}
P+Q & L & M & 0 & \frac{i}{\sqrt{2}} L & -i \sqrt{2} M \\
L^{\dagger} & P-Q & 0 & M & -i \sqrt{2} Q & i \sqrt{\frac{3}{2}} L \\
M^{\dagger} & 0 & P-Q & -L & i \sqrt{\frac{3}{2}} L^{\dagger} & -i \sqrt{2} Q \\
0 & M^{\dagger} & -L^{\dagger} & P+Q & -i \sqrt{2} M^{\dagger} & -\frac{i}{\sqrt{2}} L^{\dagger} \\
-\frac{i}{\sqrt{2}} L^{\dagger} & i \sqrt{2} Q & i \sqrt{\frac{3}{2}} L & i \sqrt{2} M & P+\Delta(z) & 0 \\
i \sqrt{2} M^{\dagger} & -i \sqrt{\frac{3}{2}} L^{\dagger} & i \sqrt{2} Q & \frac{i}{\sqrt{2}} L & 0 & P+\Delta(z)
\end{array}\right),
$$


which is written here in the spin-orbit-coupled basis $|\mu\rangle=\left|J, J_{z}\right\rangle$, which can also be expressed in the LuttingerKohn $\Gamma_{15}$ zone-center $p$ states $|X\rangle,|Y\rangle$, and $|Z\rangle$, and the spin- $\frac{1}{2}$ spinors $|\uparrow\rangle,|\downarrow\rangle$ :

$$
\begin{aligned}
& \left|\frac{3}{2},+\frac{3}{2}\right\rangle=\frac{1}{\sqrt{2}}(|X\rangle+i|Y\rangle)|\uparrow\rangle, \\
& \left|\frac{3}{2},+\frac{1}{2}\right\rangle=\frac{i}{\sqrt{6}}[(|X\rangle+i|Y\rangle)|\downarrow\rangle-2|Z\rangle|\uparrow\rangle], \\
& \left|\frac{3}{2},-\frac{1}{2}\right\rangle=\frac{1}{\sqrt{6}}[(|X\rangle-i|Y\rangle)|\uparrow\rangle-2|Z\rangle|\downarrow\rangle], \\
& \left|\frac{3}{2},-\frac{3}{2}\right\rangle=\frac{i}{\sqrt{2}}(|X\rangle-i|Y\rangle)|\downarrow\rangle, \\
& \left|\frac{1}{2},+\frac{1}{2}\right\rangle=\frac{1}{\sqrt{3}}[(|X\rangle+i|Y\rangle)|\downarrow\rangle+|Z\rangle|\uparrow\rangle], \\
& \left.\left|\frac{1}{2},-\frac{1}{2}\right\rangle=\frac{i}{\sqrt{3}}[(-|X\rangle+i|Y\rangle) \uparrow\rangle+|Z\rangle|\downarrow\rangle\right] .
\end{aligned}
$$

Hereby, we used the abbreviations

$$
\begin{gathered}
P=\frac{\hbar^{2}}{2 m_{0}} \gamma_{1} k^{2}, \\
Q=\frac{\hbar^{2}}{2 m_{0}} \gamma_{2}\left(k_{x}^{2}+k_{y}^{2}-2 k_{z}^{2}\right), \\
L=-i \sqrt{3} \frac{\hbar^{2}}{2 m_{0}} \gamma_{3}\left(k_{x}-i k_{y}\right) k_{z}, \\
M=\sqrt{3} \frac{\hbar^{2}}{2 m_{0}}\left[\gamma_{2}\left(k_{x}^{2}-k_{y}^{2}\right)-2 i \gamma_{3} k_{x} k_{y}\right], \\
\gamma_{1}, \gamma_{2}, \gamma_{3}: \text { Luttinger parameter, } \\
\Delta=E_{\Gamma_{8}}-E_{\Gamma_{7}}: \text { spin-orbit splitting. }
\end{gathered}
$$

In the kinetic operators $T_{\mu, \mu^{\prime}}$, we replace the quasimomentum $\hbar \vec{k}$ by the corresponding operator $-i \hbar \vec{\partial}$. To obtain a Hermitian Hamiltonian even with spatially varying Luttinger parameters $\gamma_{1,2,3}(z)$, we symmetrize the operators in the following way: ${ }^{3}$

$P(z) \partial_{z} \rightarrow \frac{1}{2}\left[\partial_{z} P(z)+P(z) \partial_{z}\right], \quad P(z) \partial_{z} \partial_{z} \rightarrow \partial_{z} P(z) \partial_{z}$.

$P(z)$ represents in $T_{\mu, \mu^{\prime}}$, a combination of the Luttinger parameters $\gamma_{1,2,3}$. This procedure is not unequivocal, as is discussed, e.g., by Nojima. ${ }^{55}$ Diagonalizing this Hamiltonian in the product basis of the envelope functions and the spinorbit-coupled Wannier states of the valence band $|\mu\rangle$, e.g., solving Eq. (3), gives the subband wave functions $\Sigma_{\mu} \Psi_{\mu}^{n}(\vec{k}, z)|\mu\rangle$ for every subband state $n$ and quasimomentum $\hbar \vec{k}$.

We now consider the optical transition matrix element $P_{\vec{\epsilon}}$ between the $l$ th $\Gamma$ conduction subband $\Psi^{l}(\vec{k}, z)|0,0\rangle\left|s_{\mathrm{e}}\right\rangle$ and the $m$ th valence subband $\Sigma_{\mu} \Psi_{\mu}^{m}(\vec{k}, z)|\mu\rangle$ for the light polarization $\vec{\epsilon}$. It can be written down using the representation of the spin-orbit coupled basis $|\mu\rangle=\left|J, J_{z}\right\rangle$ in the un- coupled basis $(|X\rangle,|Y\rangle,|Z\rangle)\left|s_{h}\right\rangle$ :

$$
\begin{aligned}
& P_{\dot{\epsilon}}^{l, m}(\vec{k}) \propto \sum_{\mu=1}^{6} \vec{\epsilon} \cdot\left\langle s_{\mathrm{e}}\right|\left\langle 0,0\left|\Psi^{l}(\vec{k}, z)\right| \vec{\partial}\left|\Psi_{\mu}^{m}(\vec{k}, z)\right| \mu\right\rangle \\
& =\sum_{\mu=1}^{6}\left(\frac{L_{z}}{N_{z} n_{z}=0} \Psi^{N_{z}-1} \Psi^{l}\left(\vec{k}, \frac{n_{z}}{N_{z} L_{z}}\right) \cdot \Psi_{\mu}^{m}\left(\vec{k}, \frac{n_{z}}{N_{z} L_{z}}\right)\right) \\
& \underbrace{}_{\text {envelope overlap }} \\
& \times\left(\sum_{s_{h}=-1 / 2}^{1 / 2} \delta\left(s_{e}, s_{h}\right) \sum_{P \in\{X, Y, Z\}} C_{s_{h}, P}^{\mu} \vec{\epsilon} \cdot\langle S|\vec{\partial}| P\rangle\right) . \\
& \text { dipole matrix element }
\end{aligned}
$$

Here, $C_{s_{\mathrm{h}}, P}^{\mu}$ are the coefficients relating the spin-orbitcoupled basis $|\mu\rangle$ to the uncoupled basis $|P\rangle\left|s_{h}\right\rangle$, as given in Eq. (5). The momentum matrix elements $\left\langle S\left|\partial_{x}\right| X\right\rangle=\left\langle S\left|\partial_{y}\right| Y\right\rangle=\left\langle S\left|\partial_{z}\right| Z\right\rangle$ are given in Ref. 56, and all other combinations vanish.

In order to calculate the band-to-band absorption, we determine the subband states and energies on an equidistant grid of the quasimomentum $\hbar \vec{k}$ within the first miniBrillouin-zone of the superlattice. To determine the density of states (DOS) in energy space $D(E)$, we cut the energy axis into equidistant $\Delta E$ segments. The same is done with the quasimomentum space, in which the DOS is constant. By adding the DOS of every cube in quasimomentum space to the corresponding energy segment of $D(E)$, we obtain the DOS in the energy space. In the [113] growth direction, we can simplify this calculation only by the symmetry of the dispersion due to Kramer's degeneracy.

To obtain sufficient statistics, we divide the miniBrillouin-zone into millions of cubes. The corresponding eigenenergies are obtained by a three-dimensional spline interpolation between the calculated eigenenergies at the grid points in the quasimomentum space, including the correct boundary conditions at the zone center and the zone edge. To obtain the combined DOS, we use the same procedure, but we also interpolate the optical transition matrix elements for the three polarization directions of light.

\section{B. $(13 / 10),(8 / 8)$, and (6/4) superlattices}

We now present numerical results for three SL's with different GaAs/AlAs thicknesses of $3.7 \mathrm{~nm} / 2.8 \mathrm{~nm}, 2.3 \mathrm{~nm} /$ $2.2 \mathrm{~nm}$, and $1.7 \mathrm{~nm} / 1.1 \mathrm{~nm}$, corresponding to our samples $(13 / 10),(8 / 8)$, and $(6 / 4)$, respectively (Table I). The sample labels give the GaAs/AlAs thicknesses in (001) ML's (0.283 $\mathrm{nm})$. The GaAs/AlAs material parameters used in the calculations are listed in Table II. For each SL, we compare the dispersions obtained for 0 and 6-ML APC to illustrate the maximum impact of the interface corrugation on the subband dispersions and interband transitions. The three samples cho- 
TABLE I. Sample structural parameters for the GaAs/AlAs SL samples used in this paper. The labels are chosen to represent the GaAs/AlAs thicknesses in [001] ML $(0.283 \mathrm{~nm})$. The given data are the nominal (nom.), the RHEED oscillation calibration (RHEED), and the high-resolution $\mathrm{x}$ ray characterization ( $\mathrm{x}$ ray).

\begin{tabular}{lccc}
\hline $\begin{array}{l}\text { Sample } \\
\text { name }\end{array}$ & $\begin{array}{c}\text { GaAs }(\mathrm{nm}) \\
\text { nom./RHEED/x ray }\end{array}$ & $\begin{array}{c}\text { AlAs }(\mathrm{nm}) \\
\text { nom./RHEED/x ray }\end{array}$ & $\begin{array}{c}\text { Number of } \\
\text { periods }\end{array}$ \\
\hline$(6 / 4)$ & $1.70 /-/ 1.75$ & $1.13 /-/ 1.11$ & 50 \\
$(7 / 5)$ & $3.34 /-/-$ & $1.41 /-/-$ & 50 \\
$(8 / 8)$ & $2.26 /-/ 2.3$ & $2.26 /-/ 2.2$ & 180 \\
$(10 / 10)$ & $2.83 /-/ 3.0$ & $2.83 / 2.8$ & 140 \\
$(9 / 5)$ & $2.55 /-/ 2.6$ & $1.41 / 1.4$ & 180 \\
$(9 / 10)$ & $2.55 /-/ 2.6$ & $2.83 / 2.8$ & 180 \\
$(9 / 14)$ & $2.55 /-/ 2.5$ & $3.96 / 4.0$ & 180 \\
$(10 / 5)$ & $2.83 / 2.96 /-$ & $1.41 / 1.4 /-$ & 50 \\
$(12 / 7)$ & $3.34 / 3.5 /-$ & $1.98 / 1.9 /-$ & 50 \\
$(12 / 5)$ & $3.34 /-/ 3.05$ & $1.41 /-/ 1.81$ & 50 \\
$(13 / 10 a)$ & $3.68 / 3.8 /-$ & $2.83 / 2.8 /-$ & 50 \\
$(13 / 10 b)$ & $3.68 /-/ 3.4$ & $2.83 /-/ 2.6$ & 100 \\
$(14 / 5)$ & $3.96 /-/ 3.8$ & $1.41 /-/ 1.3$ & 50 \\
\hline \hline
\end{tabular}

sen cover the range from a case nearly unaffected by the corrugation (13/10) to a quantum-wire array structure (6/4).

The subband dispersions of the $\Gamma$-point valence subbands and the $\Gamma$ - and $X_{z}$-point conduction subbands in the (13/10) SL are displayed in Fig. 2(a) for flat interfaces. Here the lowest electronic states in adjacent GaAs layers are nearly decoupled, as is evident from the flat dispersion along the [113] direction $\left(k_{z}\right)$. The in-plane dispersions $\left(k_{\|}\right)$are given

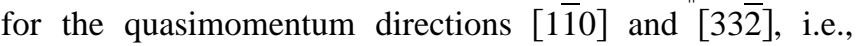
along and perpendicular to the direction of possible corrugation. The $\Gamma 1$ subband dispersions in these two in-plane directions are equal due to the isotropy of the $\Gamma$ conductionband minimum in the applied approximation. The $X_{z}$ conduction-band minimum has an anisotropic effective mass, which is large along the [001] direction, resulting in an $X_{z} 1$ subband dispersion that is steeper along the [110] direc-

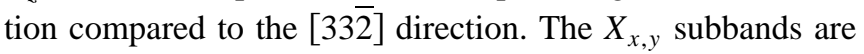
not shown here because they are energetically above the $X_{z} 1$ subband, and are thus not important for the optical properties of the considered short-period SL's. The valence sub-

TABLE II. Material parameters of GaAs and AlAs used in the calculations (Ref. 69).

\begin{tabular}{lcccc}
\hline \hline Symbol & Parameter & Unit & GaAs & AlAs \\
\hline$E_{g}(\Gamma-\Gamma)$ & direct gap & $(\mathrm{eV})$ & 1.519 & 3.1 \\
$E_{g}(\Gamma-X)$ & indirect gap & $(\mathrm{eV})$ & 1.900 & 2.17 \\
$\Delta E_{c} / \Delta E_{v}$ & band offset & - & \multicolumn{2}{c}{$67 / 33$} \\
$m_{e}$ & $\Gamma$ electron mass & $\left(m_{0}\right)$ & 0.067 & 0.15 \\
$m_{X}^{\|}$ & $X$ longitudinal mass & $\left(m_{0}\right)$ & 1.3 & 1.1 \\
$\mathrm{~m}_{\mathrm{X}}^{\perp}$ & $X$ transversal mass & $\left(m_{0}\right)$ & 0.23 & 0.19 \\
$\gamma_{1}$ & Luttinger & - & 7.1 & 3.76 \\
$\gamma_{2}$ & parameters & - & 2.02 & 0.90 \\
$\gamma_{3}$ & & - & 2.91 & 1.42 \\
$\Delta_{\text {so }}$ & spin-orbit splitting & $(\mathrm{eV})$ & 0.34 & 0.275 \\
\hline \hline
\end{tabular}
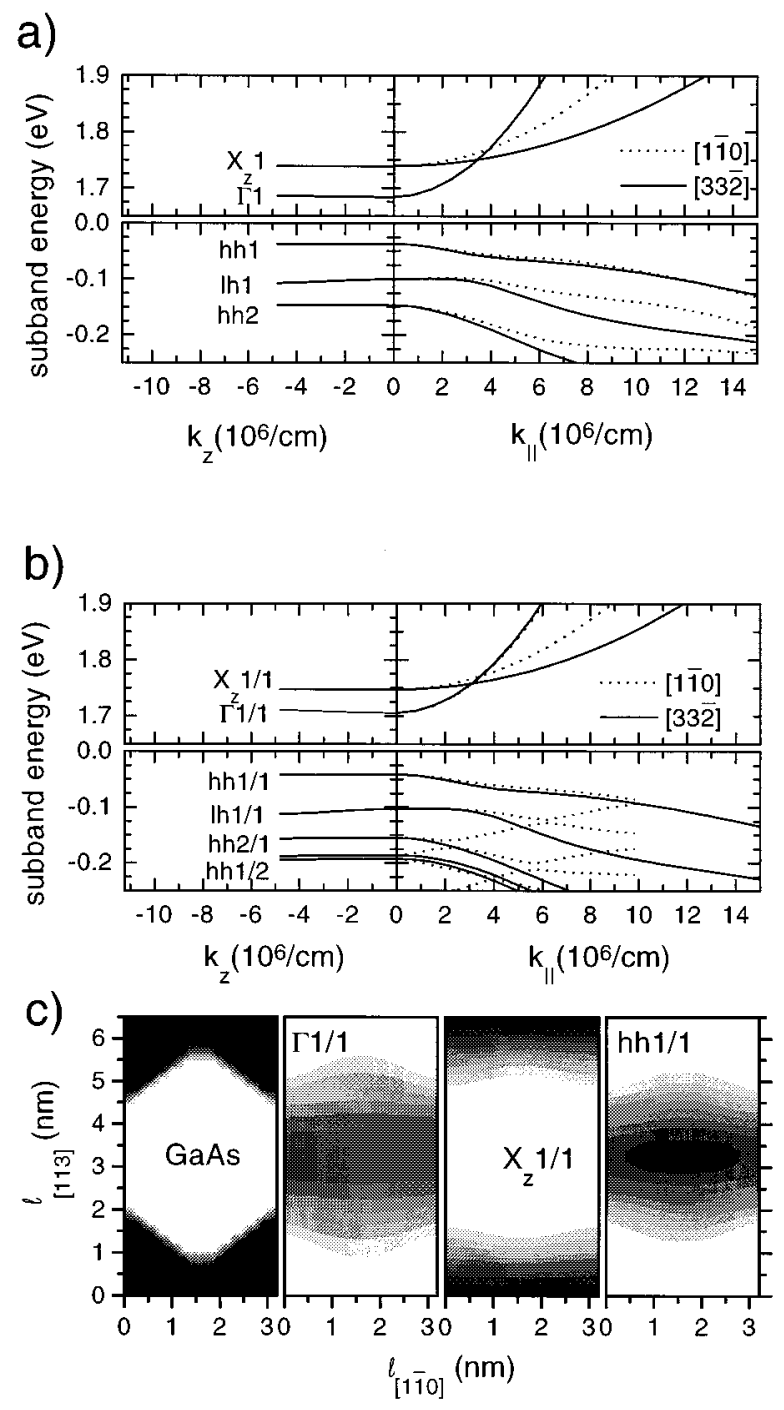

FIG. 2. Calculated subband states and dispersions of the $\Gamma$ and $X_{z}$ conduction-band minima and of the $\Gamma$ valence-band maximum in a (113)-grown (13/10) SL. (a) and (b) Subband dispersions in the presence of 0 and 6-ML APC, respectively. The quasimomentum is given relative to the considered band extremum. The in-plane dis-

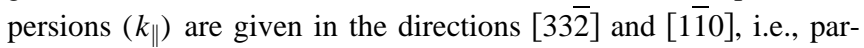
allel and orthogonal to the corrugation. (c) Real-space distribution of the squared envelope functions of the lowest subband states in the presence of 6-ML APC. The contours are given on a linear scale from 0 to 3.5, with the integral normalized to 1 within the displayed unit cell. The GaAs/AlAs distribution is given in the leftmost contour for comparison.

bands hh1, lh1, and hh2 are a mixture of of heavy-hole, light-hole, and split-off bands, but they are labeled after their dominant part. The in-plane dispersions of these subbands show an anticrossing behavior caused by the mixing of the different valence bands. They additionally exhibit an in-

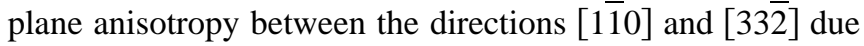
to the intrinsic properties of GaAs and AlAs, i.e., the zincblende symmetry. This anisotropy, present even in SL's with flat interfaces, is obviously not a consequence of an interface corrugation.

Including a 6-ML APC, the subband dispersions [Fig. 2(b)] show a backfolding of the Brillouin zone along 

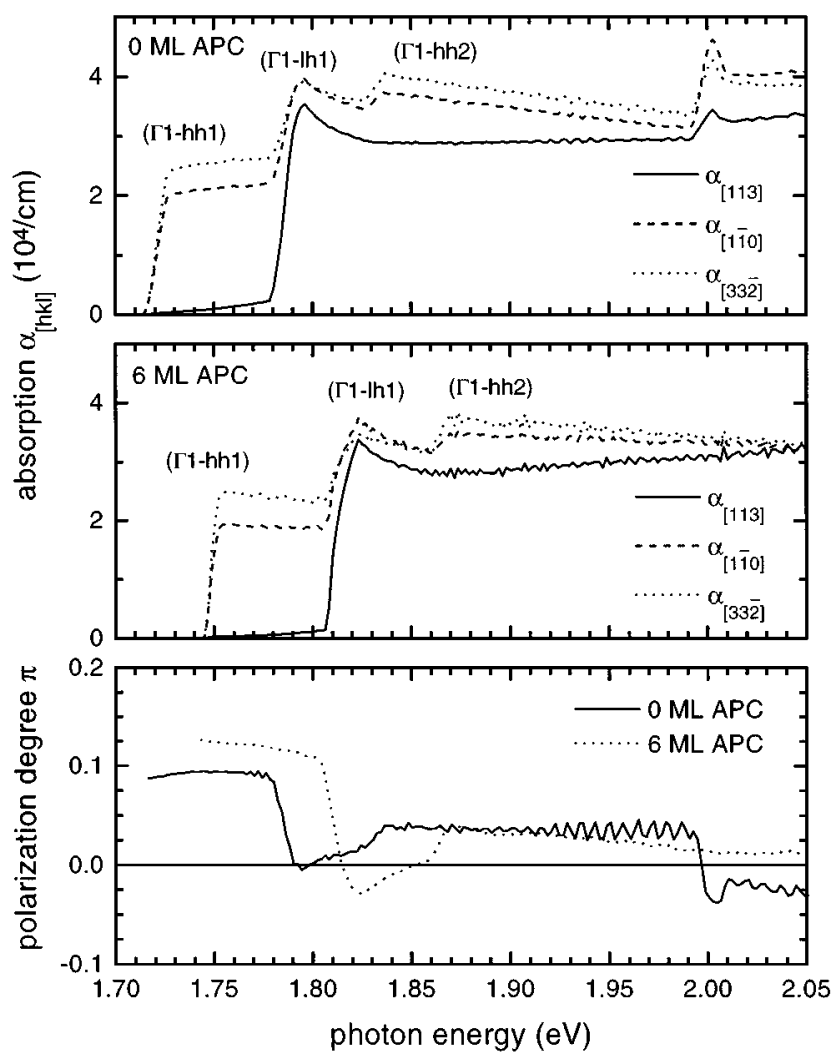

FIG. 3. Calculated absorption $\alpha_{[h k l]}$ in a (113)-grown (13/10) GaAs/AlAs SL with 0- and 6-ML APC for the three principal light polarizations, as indicated. The transitions are labeled. The lowest section shows the corresponding absorption PD $\pi=\left(\alpha_{[33 \overline{2}]}-\alpha_{[1 \overline{10]}}\right) /\left(\alpha_{[33 \overline{2}]}+\alpha_{[1 \overline{1} 0]}\right)$ for light propagating along the growth direction [113].

the $[1 \overline{1} 0]$ direction into a mini-Brillouin-zone, and the splitting of the dispersions at the corresponding zone edge and zone center due to the periodic potential introduced by the corrugation. This gives rise to an additional quantum number

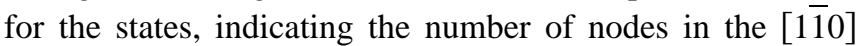
direction. Due to the large period of the (13/10) SL, the effect of the corrugation is small, and has only a minor influence on the dispersion. The hh1 effective mass is 0.21 in the $[1 \overline{1} 0]$ direction and 0.16 in the $[33 \overline{2}]$ direction, the same as for 0-ML APC. The subband envelope functions [Fig. 2(c)] show the influence of the corrugation by their modulation along the $[1 \overline{1} 0]$ direction. The modulation is weak compared to the modulation along the growth direction [113], and much smaller than necessary for a quantum wire behavior.

The calculated absorption $\alpha_{[h k l]}$ and the PD $\pi=\left(\alpha_{[33 \overline{2}]}-\alpha_{[1 \overline{1} 0]}\right) /\left(\alpha_{[33 \overline{2}]}+\alpha_{[1 \overline{1} 0]}\right)$ are shown in Fig. 3 for the principal light polarization directions [113], [110], and [332 $]$. The polarization directions are established by the growth direction in the flat structure, and are the same for the flat as well as the corrugated structure due to the orientation of the corrugation along one of the main polarization directions of the flat sample. The absorption consists of the ( $\Gamma 1-\mathrm{hh} 1),(\Gamma 1-\mathrm{lh} 1)$, and $(\Gamma 1-\mathrm{hh} 2)$ transition. The ( $\Gamma 1-\mathrm{hh} 1)$ transition is only active for in-plane polarization, while the
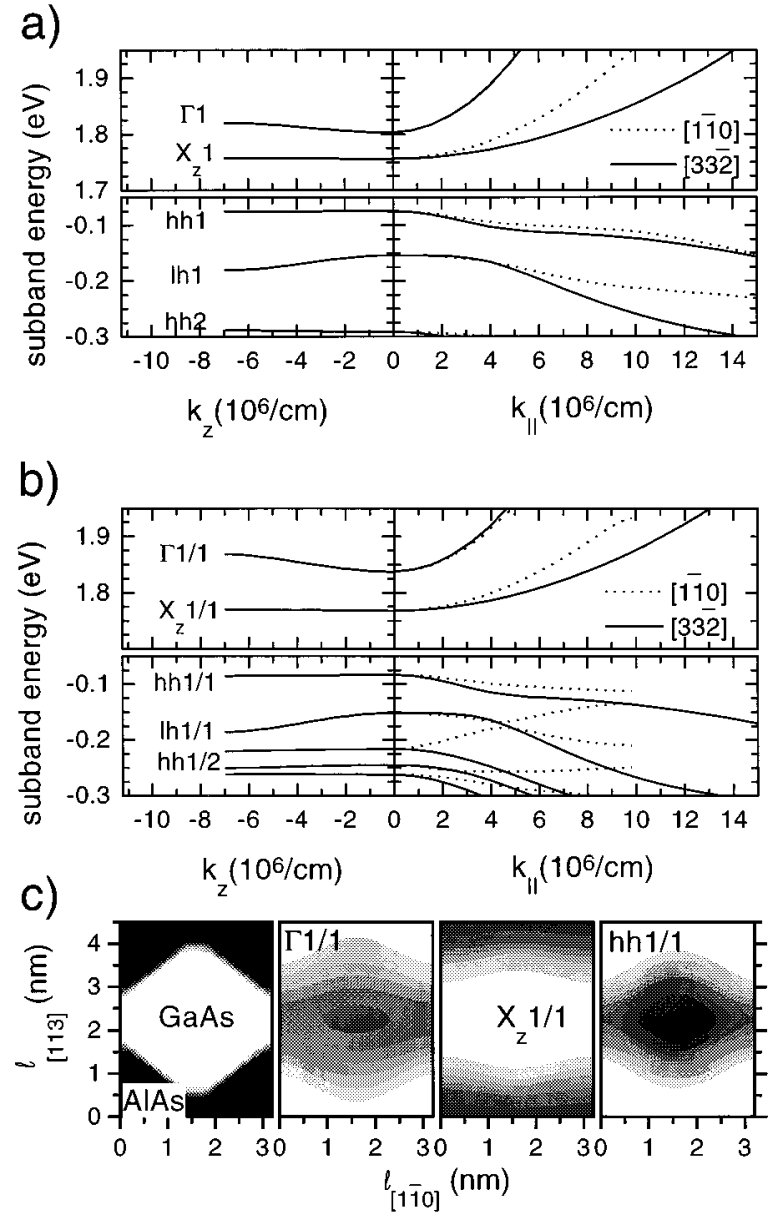

FIG. 4. As Fig. 2, but for a (8/8) SL.

( $\Gamma 1-\mathrm{lh} 1)$ transition is strongest for the polarization along the growth direction. The ( $\Gamma 1-\mathrm{hh} 2)$ transition is weak due to the reduced wave function overlap integral. This holds for both interface structures. The PD of the ( $\Gamma 1-\mathrm{hh} 1)$ transition intrinsic to the [113] growth direction is enhanced by the 6-ML APC, as is shown in the lowest graph of Fig. 3. The corrugation additionally leads to a small overall shift of the transitions to higher energies.

The corresponding results for the (8/8) SL are given in Figs. 4 and 5. In this SL, the subband splitting is considerably larger than in the (13/10) case, and the miniband dispersion along the growth direction reaches roughly $20 \mathrm{meV}$ for the $\mathrm{lh} 1$ and $\Gamma 1$ subbands. This leads to a broadening of the absorption structures at the subband edges (Fig. 5). The corrugation has a larger influence in this SL, shown by the stronger splitting of the subband dispersions in the [1]0] direction at the edge of the mini-Brillouin-zone [Fig. 4(b)]. The corrugation increases the effective mass of the $\Gamma 1$ subband in the [110] direction by $3 \%$, and that of the hh1 subband by $18 \%$. The subband envelope functions [Fig. 4(c)] are more strongly modulated by the corrugation due to the smaller period length.

The PD of the (Г1-hh1) transition (Fig. 5) is stronger than in the (13/10) SL even in the absence of a corrugation due to the stronger contribution of the split-off valence band to the hh1 subband at the higher hh1 quantization energy in this SL. ${ }^{57}$ The 6-ML APC nearly doubles this PD. 


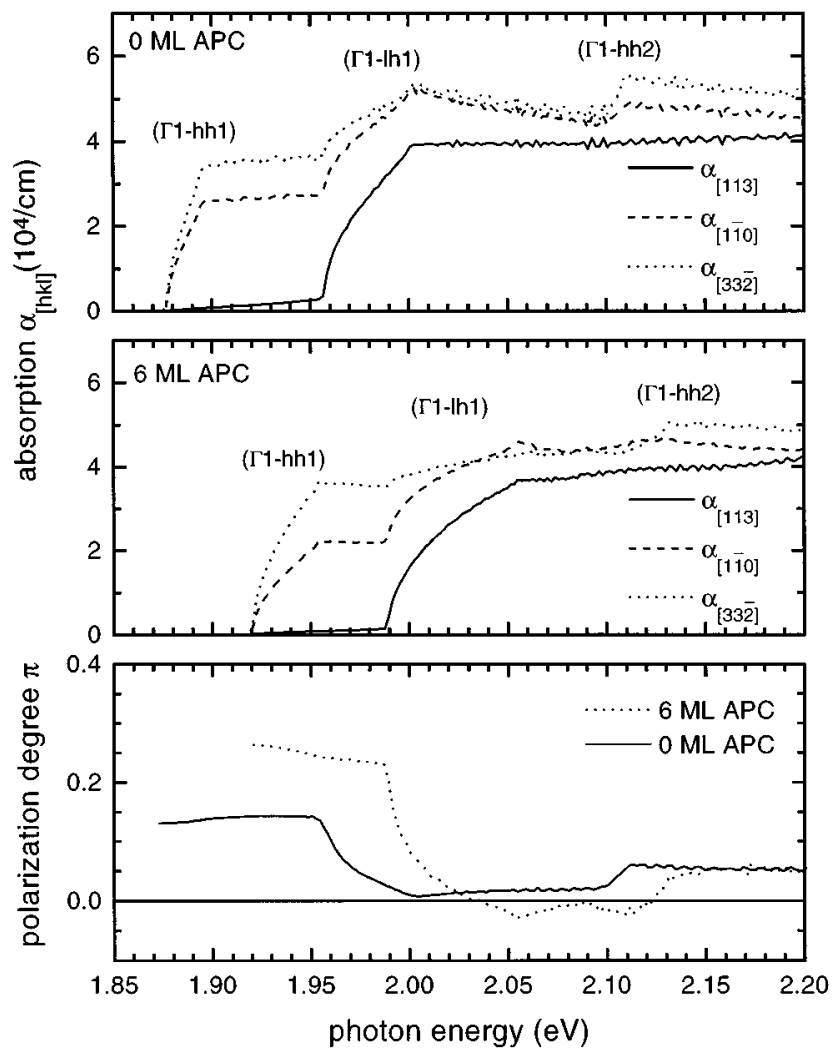

FIG. 5. As Fig. 3, but for a (8/8) SL.

The impact of a 6-ML APC on the subband dispersions is again stronger for the (6/4) SL, for which the calculated results are shown in Figs. 6 and 7. The small period results in a large miniband width especially for the $\Gamma 1$ and $\mathrm{lh} 1$ subbands [Fig. 6(a)]. Including 6-ML APC [Fig. 6(b)], the miniband width along the [1 $\overline{1} 0]$ direction becomes comparable to the width in the [113] growth direction, revealing the qualitative change from a SL into a quantum-wire array. This is especially evident for the hh1 subband, having a smaller miniband width along the [1실 direction than along the growth direction. The effective-mass enhancement in the [110] direction amounts to $20 \%$ for the $\Gamma 1$ subband and to $530 \%$ for the hh1 subband. The shape of the structure in real space is nearly rhombic [Fig. 6c], and the modulations of the subband envelope functions are comparable in the growth and the corrugation direction.

The absorption of the SL is governed by the ( $\Gamma 1-\mathrm{hh} 1)$ and (Г1-lh1) transitions (Fig. 7). The large miniband widths of both transitions lead to a smooth onset of the subband absorption. The PD of the ( $\Gamma 1-\mathrm{hh} 1)$ transition is strongly increased by the corrugation from 0.17 to 0.75 , and is thus dominated by the corrugation effect. We therefore conclude that a 6-ML APC leads to strong anisotropies of the electronic properties in the (6/4) SL, but a one-dimensional behavior of the density of states is not achieved due to the large miniband widths.

\section{Dependence on the period length}

After the discussion of these three examples, we present the calculated period length dependence of the subband en-
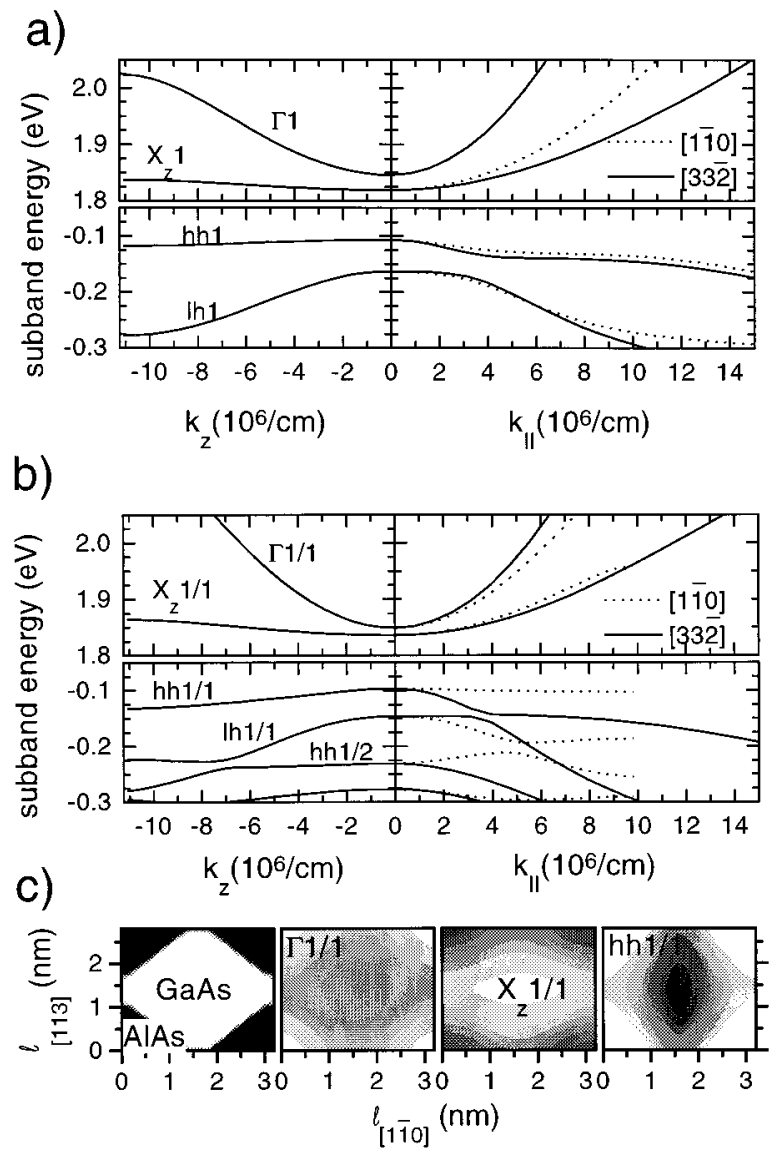

FIG. 6. As Fig. 2, but for a (6/4) SL.

ergies and the optical anisotropy including the interface corrugation. This dependence is displayed in Fig. 8 for the $\Gamma 1, X_{z} 1$, and hh1 subband energies and the corresponding transition energies for (001)- and (113)-grown symmetric SL's with 0- and 6-ML APC.

Changing the growth direction from [001] to [113], the $X_{z} 1$ quantization energy increases due to the deviation of the quantization axis from the longitudinal direction of the effective-mass ellipsoid. The $\Gamma 1$ subband energy remains unchanged due to the isotropy of the $\Gamma$ conduction-band minimum. Together, this shifts the $\Gamma-X$ crossing to smallerperiod lengths. The hh1 quantization energy is decreased due to the higher mass in growth direction introduced by the change in valence-band mixing. ${ }^{33}$ This results in a redshift of the $(\Gamma 1-h h 1)$ transition.

The transition-energy changes due to the interface corrugation [Fig. 8(d)] as a function of period length show a maximum. This can be understood by considering the influence of a varying film thickness on the mean subband energy. Introducing a small relative variation $\Delta L$ of the layer thickness $L$ leads to a shift $\Delta \mathrm{E}$ of the mean energy proportional to the second derivative of the subband energy $E$ with respect to $L$ :

$$
\Delta E=\frac{1}{2} \frac{\partial^{2} E}{\partial L^{2}}\left\langle\Delta L^{2}\right\rangle \quad \text { with }\langle\Delta L\rangle=0 .
$$

The effect of the corrugation is therefore most pronounced at the maximum of the second derivative $\partial^{2} E / \partial L^{2}$ of the sub- 


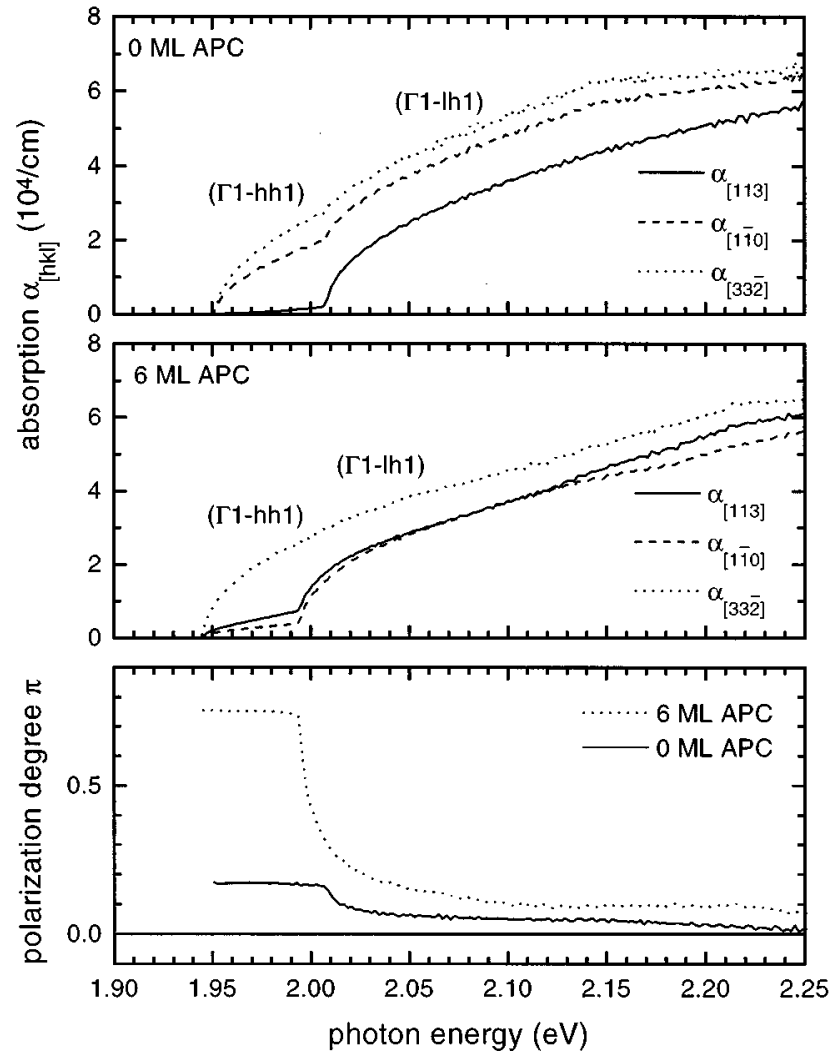

FIG. 7. As Fig. 3, but for a (6/4) SL.

band energies with respect to the period length. Using the calculated energies [Fig. 8(c)], we find the maximum at about 5-nm period length due to the penetration of the envelope functions into the barriers. The resulting shifts obtained with Eq. (8) [curves in Fig. 8(d)] are only in qualitative agreement with the numerically calculated shift [symbols in Fig. 8(d)], especially for the light particles, e.g., the $\Gamma 1$ subband. This is because we have neglected the kinetic energy in the corrugation direction [110] that is necessary for the wave functions to follow the corrugation. Taking this into account, the wave functions only partly penetrate into the wide regions of the structure, thereby minimizing the sum of the kinetic energies in the [1 $\overline{1} 0]$ direction and in the [113] growth direction. This qualitative argument is illustrated by the calculated envelope functions [Figs. 2(c), 4(c), and 6(c)], which do not extend fully into the wide regions of the structure.

The second prediction of Eq. (8) is the quadratic dependence of $\Delta E$ on the corrugation height. The numerical results on the corrugation height dependence of the PD are shown in Fig. 9 for the ( $\Gamma 1$-hh1) transition in a (8/8) SL. The nonzero PD for flat interfaces is increased by the corrugation with the expected quadratic behaviour. The in-phase corrugation (IPC) has, as is also expected from intuition, less effect on the PD than the APC. One should note that Eq. (8) predicts no effect of an IPC and is thus limited to a qualitative description.

Let us now discuss the calculated PD's of the lowest two transitions $(\Gamma 1-\mathrm{hh} 1)$ and $(\Gamma 1-\mathrm{lh} 1)$ as function of the period length for symmetric SL's [Fig. 10(a)]. SL's with flat interfaces (solid curve) show an increasing PD with decreasing

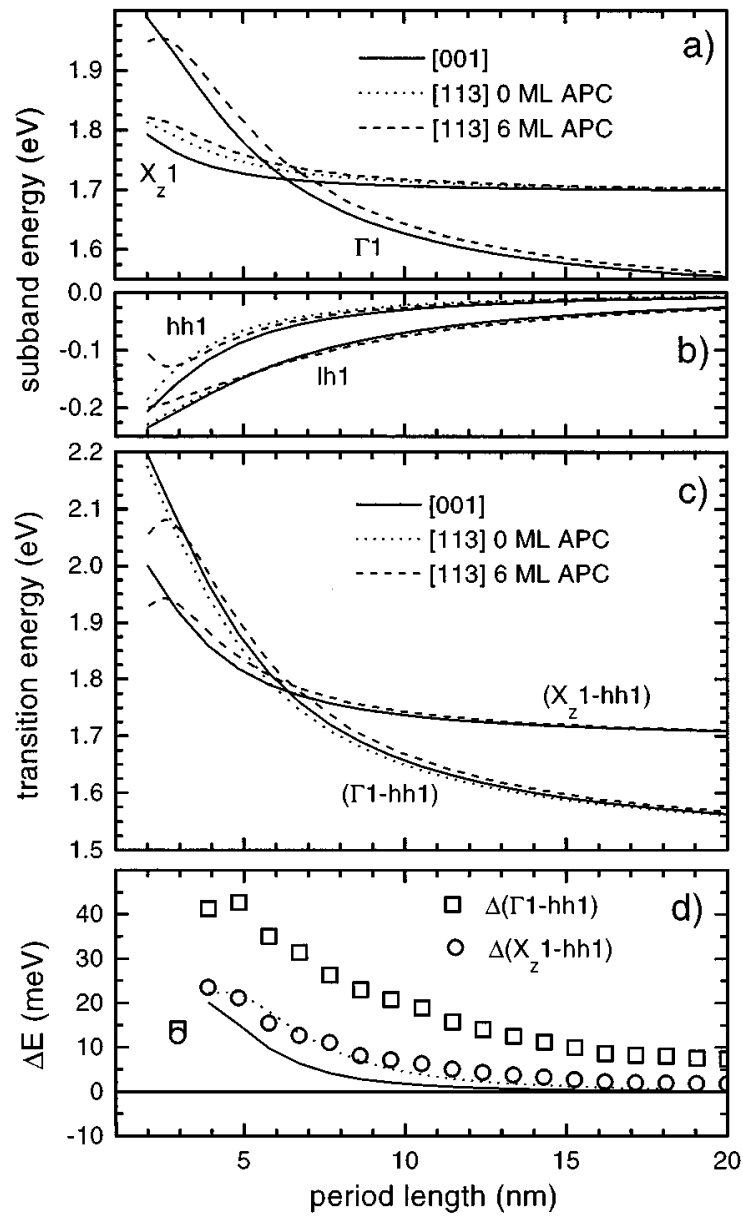

FIG. 8. Calculated energies in symmetric GaAs/AlAs SL's depending on the period length. The results for (001)-grown SL's with 0-ML APC and for (113)-grown SL's for 0- and 6-ML APC are shown. (a) $\Gamma 1$ and $X_{z} 1$ conduction subbands; (b) hh1 and lh1 valence subbands; (c) ( $\Gamma 1-\mathrm{hh} 1)$ and $\left(X_{\mathrm{z}} 1-\mathrm{hh} 1\right)$ transitions; (d) energy change due to 6-ML APC. The lines in show the energy change calculated with Eq. (8). Dotted: $\Delta(\Gamma 1-\mathrm{hh} 1)$. Solid: $\Delta\left(X_{z} 1-\mathrm{hh} 1\right)$.

period length due to the increasing admixture of the split-off valence band. ${ }^{35,58}$ This increase is, for period lengths larger than $4 \mathrm{~nm}$, nearly proportional to $1 / L$, as is shown in Fig. 10(b) for the ( $\Gamma 1-\mathrm{hh} 1)$ transition. The $1 / L^{2}$ dependence predicted by first-order perturbation theory for infinitely high barriers $^{57}$ is not applicable here due to the finite barrier height, leading to a stronger admixture of the split-off hole. ${ }^{59}$

The corrugation leads to an enhancement of the PD which is proportional to $1 / L^{2}$ at large period lengths [dashed and dotted lines in Fig. 10(b)]. Consequently, even 6-ML APC has a significant influence on the PD only for period lengths below $10 \mathrm{~nm}$. Due to the quadratic dependence on the corrugation height, a 3-ML APC has a significant influence only for even smaller period lengths [dashed line in Fig. 10(a)].

In summary, calculations show that the interface corrugation leads to a significant change of the subband dispersions and wave functions only for superlattices below a $10 \mathrm{~nm}$ period length. For larger period lengths, the lateral confinement is not strong enough to change the wave function on the short lateral scale of the corrugation. For short-period superlattices, the corrugation leads to an enhanced polarization anisotropy and to a larger effective mass along the cor- 


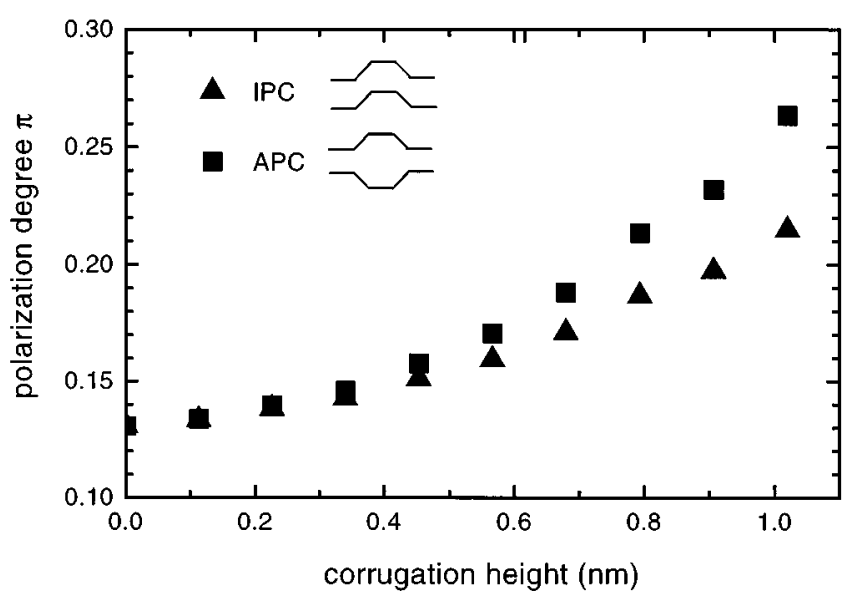

FIG. 9. Calculated PD of the ( $\Gamma 1-\mathrm{hh} 1)$ transition in a (113)grown (8/8) SL depending on the corrugation height. The results for APC (squares) and IPC (triangles) are shown.

rugation due to the modulation of the wave functions. However, a one-dimensional character of the density of states is not achieved even for the smallest periods due to the coupling of the wave functions through the barriers, leading to a large miniband width.

\section{EXPERIMENTAL RESULTS ON THE ANISOTROPY}

The calculation of the optical anisotropy, described in Sec. II, allows us to determine the corrugation height present
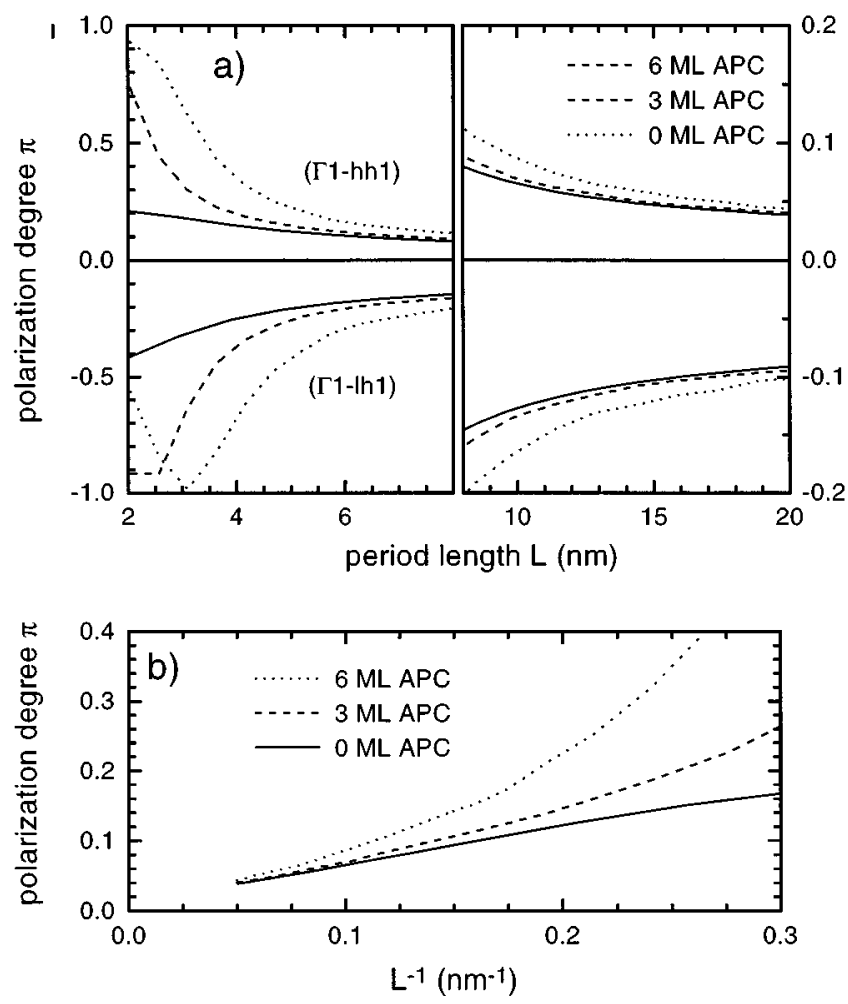

FIG. 10. (a) Calculated PD of the ( $\Gamma 1-\mathrm{hh} 1)$ and ( $\Gamma 1-\mathrm{lh} 1)$ transitions in symmetric (113)-grown SL's as a function of period length for 0-ML (solid), 3 ML (dashed), and 6-ML (dotted) APC. (b) PD of the $(\Gamma 1-h h 1)$ transition on a reciprocal period length scale. in the samples under investigation. They were grown in a Meca MBE growth chamber at $600{ }^{\circ} \mathrm{C}$ simultaneously on (001) and (113) GaAs substrates, soldered side by side on a molybdenum block. The structures of the samples are given in Table I. The growth rate was $0.7 \mu \mathrm{m} / \mathrm{h}$ for GaAs and AlAs, and the $\mathrm{As}_{4} / \mathrm{Ga}$ flux ratio was about 5. The growth on the (113) plane was monitored by RHEED at $20 \mathrm{kV}$ using incidence angles around $1^{\circ}$. At each heterointerface, the growth was interrupted for $60 \mathrm{~s}$. During growth, the RHEED pattern maintained the typical $(8 \times 1)$ reconstruction.

To determine the optical anisotropy of the ( $\Gamma 1-\mathrm{hh} 1)$ transition experimentally, we have used three different methods, namely transmission, PLE, and PL spectroscopy. The samples were mounted in a helium bath cryostat to achieve sample temperatures between 5 and $300 \mathrm{~K}$.

For the transmission measurements, the GaAs substrate was removed by selective etching. The white light source was a tungsten lamp. The polarization was analyzed using a combination of a Fresnel rhomb and a crystal polarizer in front of the spectrometer. This avoids any influence of the spectrometer polarization properties on the results.

In PLE measurements, we used the light from a xenon high-pressure lamp dispersed by a 1-m single spectrometer as excitation source. The PL was detected by a photomultiplier using a lock-in technique. In this case the crystal polarizer and the Fresnel rhomb were used to polarize the excitation light. The intensity dependence of the PL efficiency of the samples was measured separately and taken into account to calculate the absorption anisotropy from the PLE data (corrected PLE).

In the PL measurements, the samples were excited by a green He-Ne laser. The PL was dispersed by a single $27-\mathrm{cm}$ spectrometer and detected by an intensified diode array.

\section{A. Absorption}

Figure 11 shows the optical thickness $\alpha d$ and its PD of the $(8 / 8)$ sample with a removed substrate at $5 \mathrm{~K}$ lattice temperature. The optical thickness is calculated from the experimental transmission taking into account the reflection, and is shown for [33르 polarization together with the calculated optical thickness for flat interfaces [Fig. 11(a)]. The calculation overestimates the transition energies by about 50 $\mathrm{meV}$. Apart from that, there is a quantitative agreement, keeping in mind the inhomogeneous broadening in the experimental data. The PD of the optical density [Fig. 11(b)] is in agreement with the calculation for flat interfaces within the experimental errors, whereas the calculation for 6-ML APC deviates significantly. This shows that a $6 \mathrm{ML}$ APC is not expected to be present in this sample. As we saw in Sec. II, the change of the PD depends quadratically on the corrugation height, thus a 2-ML APC is not excluded by this result.

\section{B. Photoluminescence excitation}

The PL and corrected PLE of the (6/4) sample at $5 \mathrm{~K}$ is displayed in Fig. 12(a). The PLE is compared with the calculated optical density for unpolarized light and 0-ML APC. In this SL the miniband widths are large, leading to a smooth absorption onset even in the calculated data. The PLE shows a strong inhomogeneous broadening due to the small SL period length. The PL is dominated by the $\left(X_{z} 1-\mathrm{hh} 1\right)$ transition 


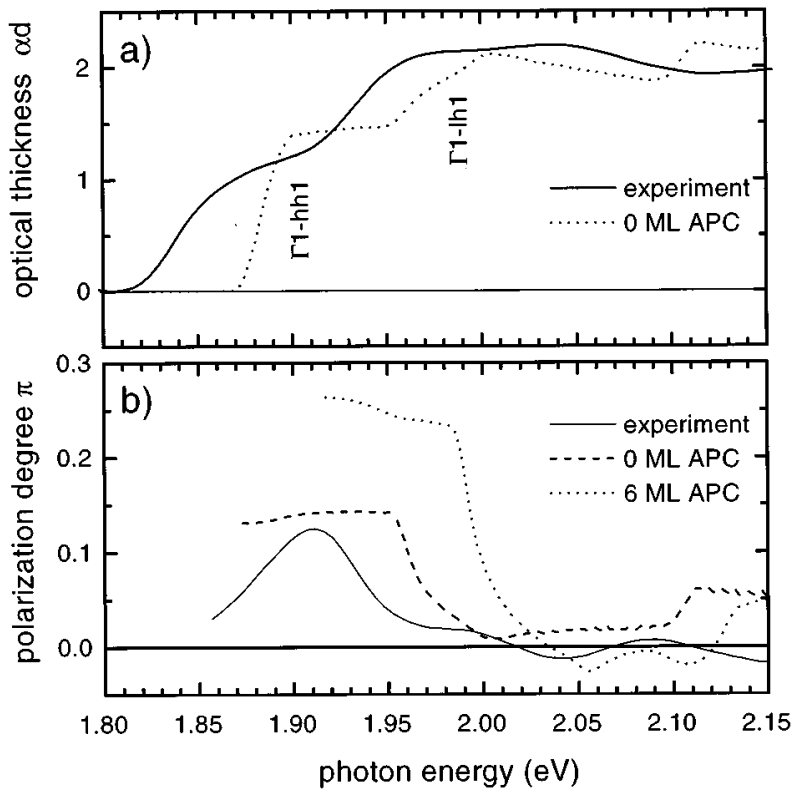

FIG. 11. Optical thickness $\alpha d$ and its PD $\pi$ calculated from the transmission of a (113)-grown (8/8) SL at 5-K lattice temperature. (a) Optical thickness for [33ㄹ] -polarized light (solid line) together with the calculated optical thickness for 0-ML APC (dotted line). (b) PD of the absorption (solid curve) together with the calculated PD for 0-ML (dashed curve) and 6-ML (dotted curve) APC.

and the corresponding phonon replica. The PD determined from PL and corrected PLE are compared in Fig. 12(b) with the calculated PD for 0 and 6-ML APC. Again, the calculation for 0-ML APC is in agreement with the experimental data, while the results for 6-ML APC strongly deviate. The

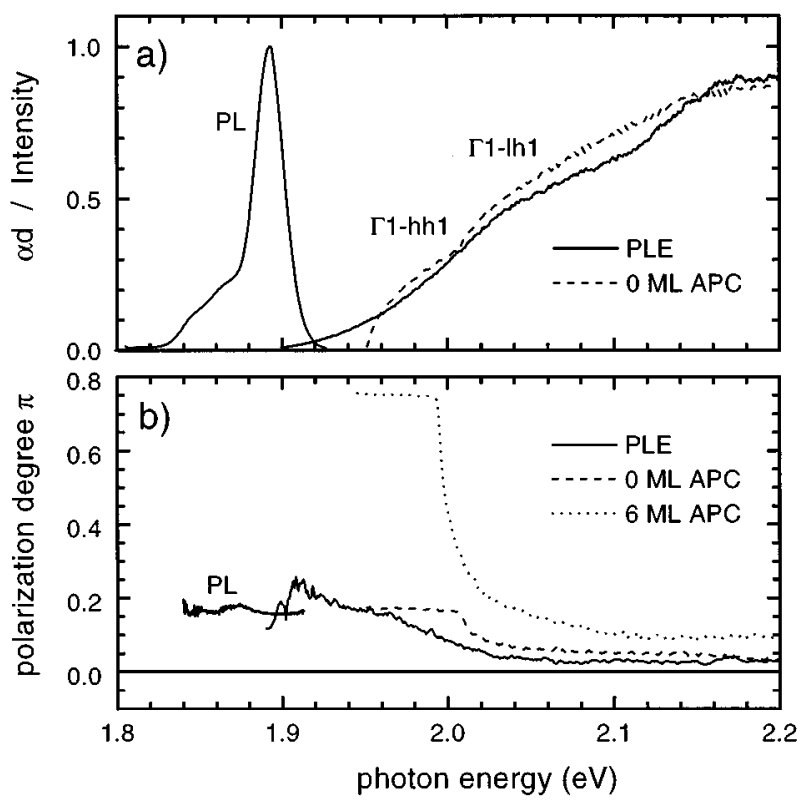

FIG. 12. PL, corrected PLE, and the corresponding PD of a (113)-grown (6/4) SL at 5-K lattice temperature. (a) Unpolarized PL, corrected PLE (solid curves), and calculated absorption for 0-ML APC (dashed curve). (b) PD of PL and corrected PLE (solid curves) together with the calculated PD for 0-ML (dashed curve) and 6-ML (dotted curve) APC.
PD of the $\left(X_{z} 1-\mathrm{hh} 1\right) \mathrm{PL}$ is equal to the PD of the PLE, involving the ( $\Gamma 1-\mathrm{hh} 1)$ transition. This is in agreement with the theoretical expectation, as both transitions involve the same valence-band states.

\section{Photoluminescence}

The PL and its PD of several SL samples with different layer thicknesses are shown in Fig. 13 together with calculated PD's for 0- and 6-ML APC. Due to the low lattice temperature of $5 \mathrm{~K}$ and an excitation density of $10 \mathrm{~mW} /$ $\mathrm{cm}^{2}$, the PL is expected to originate from localized excitonic states at the band edges. In the type-II SL's (upper row in Fig. 13), the PL is dominated by the zero-phonon and phonon-assisted $\left(X_{z} 1-\mathrm{hh} 1\right)$ transitions. The zero-phonon transition is stronger than the phonon-assisted transitions, in spite of the fact that, for the (113) growth, the SL potential does not lead to a coupling between the $X_{z} 1$ and $\Gamma 1$ subbands, ${ }^{39}$ as for the (001) growth. ${ }^{60}$ The interface disorder, which is responsible for the zero-phonon transition here, is thus expected to be stronger than in the (001)-grown samples. The latter show dominant phonon-assisted PL of the $\left(X_{x, y} 1-\mathrm{hh} 1\right)$ transition, which is, similarly, mediated only by the interface disorder ${ }^{61,62}$ The stronger interface disorder in the (113)-grown samples is also in agreement with their larger inhomogeneous width compared to equivalent (001)grown samples. ${ }^{45,13,23}$

We first concentrate on the pure type-II samples in the upper row of Fig. 13. The type-I samples will be discussed in Sec. IV. The PD of the (Xz1-hh1) PL is close to the calculated values for the ( $\Gamma 1-\mathrm{hh} 1)$ transition with 0-ML APC (solid lines in Fig. 13). Since the anisotropy arises from the hh1 subband, the calculated value for the ( $\Gamma 1-\mathrm{hh} 1)$ transition is also applicable to the $\left(\mathrm{X}_{\mathrm{z}} 1-\mathrm{hh} 1\right)$ transition. This is verified by the comparable PD of the PL and PLE in the (6/4) sample (Fig. 12).

In Fig. 14 we compare the PL PD of the type-II SL's with the calculated PD for 0-, 2, and 6-ML APC. The experimental data agree well with the calculation for $0 \mathrm{ML}$, while the calculation for 6-ML APC deviates significantly. When accounting for the uncertainties in the calculated values due to the approximations used, we cannot discard the 2-ML result from being in reasonable agreement with experiment.

These results indicate that a 6-ML APC is not present in our samples. ${ }^{29}$ On the other hand, there is evidence for an interface corrugation with a period of $3.2 \mathrm{~nm}$ along the [110] direction inside these SL's by Raman experiments, ${ }^{21,22,28}$ consistent with the RHEED image of the surface in $[33 \overline{2}]$ incidence during the growth. ${ }^{13}$ The interface corrugation within the SL's is thus most likely given by the surface reconstruction of (113) GaAs proposed by Wassermeier et al.,$^{25,26}$ showing only 2-ML corrugation height.

\section{LOCALIZED STATES}

We show in this section that the strongly anisotropic properties of the conductivity ${ }^{14}$ and the PL (Ref. 23) are most likely not due to the interface corrugation itself, but can be explained by an anisotropic interface disorder, developing in the presence of the interface corrugation. The anisotropy in the disorder is evident in STM images of the (113) GaAs 


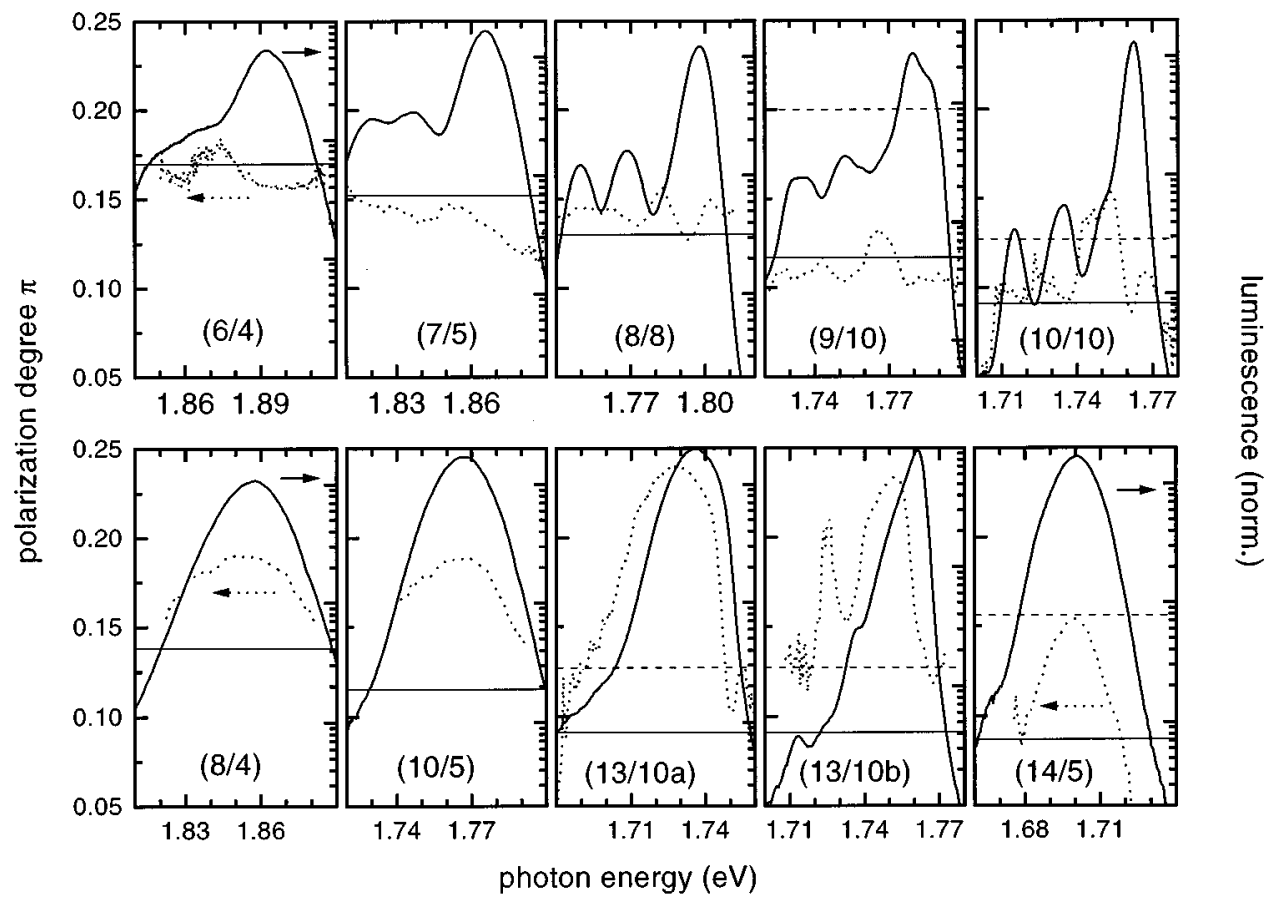

FIG. 13. PL (solid curves) and its PD (dotted curves) of several (113)-grown GaAs/AlAs SL's. The PD is given by intensity relation $\pi=\left(I_{[332]}-I_{[1[0]}\right) /\left(I_{[332]}+I_{[1\lceil 0]}\right)$. Excitation at $2.24 \mathrm{eV}$ with $10 \mathrm{~mW} / \mathrm{cm}^{2}$ at $5-\mathrm{K}$ lattice temperature. The sample labels are given (Table I). The corresponding calculated PD for 0-ML (solid horizontal lines) and 6-ML APC (dashed horizontal lines) are shown for comparison.

surface. $^{25}$ It leads to the formation of extended anisotropic potential minima, which are able to localize the band edge electronic states ${ }^{63}$ even with an interface roughness of only 2 ML . This roughness is actually present at the interfaces, as is estimated from the inhomogeneous width of the type-I PL. The reason for the anisotropic disorder is given by the surface modulation along the $[1 \overline{10}]$ direction. ${ }^{25}$ This modulation

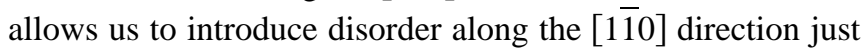
by a change of the monolayer step succession, while along

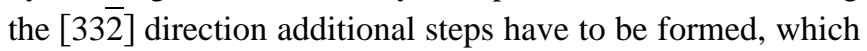
obviously involves a larger creation energy.

The PL and PLE spectra of the type-II samples (9/10), $(8 / 8)$, and $(13 / 10 \mathrm{~b})$ at $5 \mathrm{~K}$ are displayed in Fig. 15 to discuss

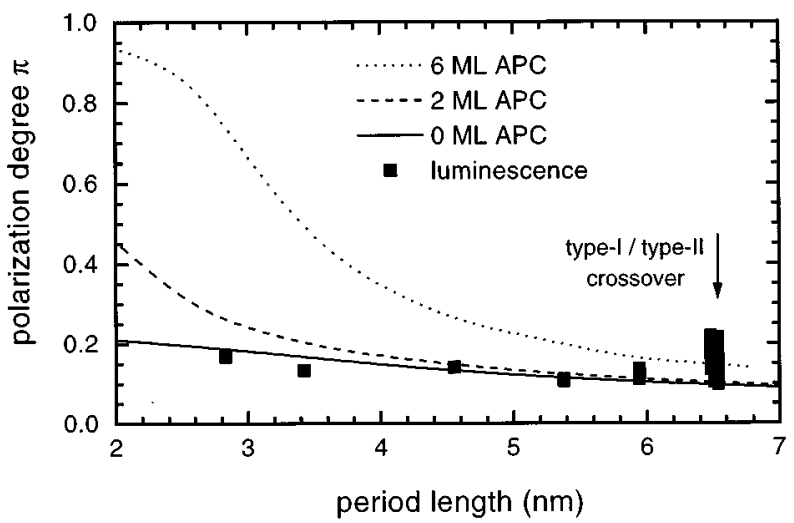

FIG. 14. Comparison between the PD of the PL (bars) and the calculated PD of the ( $\Gamma 1-\mathrm{hh} 1)$ transition in symmetric SL's with 0-ML (solid curve), 2-ML (dashed curve), and 6-ML (dotted curve) APC as function of the period length. The length of the bars give the range of the experimentally measured PD. the change of the PL properties when approaching the type-I to type-II crossover from the type-II side. The PL from the type-II samples $(9 / 10)$ and $(8 / 8)$ is dominated by the $\left(X_{z} 1-\mathrm{hh} 1\right)$ transition and its phonon replica involving the GaAs and AlAs optical phonon. The relative strength of the

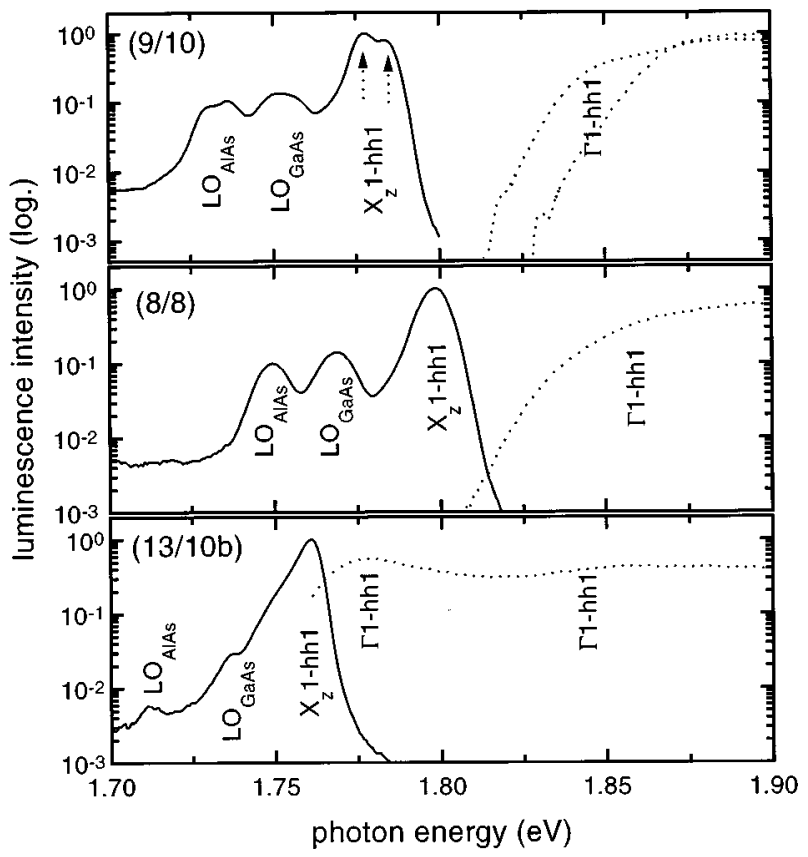

FIG. 15. PL (solid curve) and PLE (dotted curve) of samples $(13 / 10 \mathrm{~b}),(9 / 10)$, and $(8 / 8)$ at $5-\mathrm{K}$ lattice temperature and $1 \mathrm{~mW} /$ $\mathrm{cm}^{2}$ excitation intensity. The transitions are labeled. Sample (9/10) shows two different period lengths incorporated by disorder along the growth direction. 
phonon replica decreases with decreasing $\Gamma 1-X_{z} 1$ separation due to the stronger mixing between the $\Gamma 1$ and $X_{z} 1$ subband. The PLE spectra of these two samples show exponential wings below the ( $\Gamma 1-\mathrm{hh} 1)$ transition due to localized states of this transition. In the (9/10) sample, the corresponding relative density of states can be extrapolated to be about $10^{-5}$ at the $\left(X_{z} 1-\mathrm{hh} 1\right)$ transition energy. This is lower than the relative oscillator strength of the $\left(X_{z} 1-\mathrm{hh} 1\right)$ transition compared to the ( $\Gamma 1-\mathrm{hh} 1)$ transition, amounting to about $10^{-3}$, as determined by the PL decay times. Thus the PL of the localized states is not dominant in this sample at low temperatures. In the (8/8) sample, the density of the localized ( $\Gamma 1$-hh1) states at the $\left(X_{z} 1\right.$-hh1) energy is about $10^{-3}$ of the density of the extended ( $\Gamma 1-\mathrm{hh} 1)$ states. Here the PL from the localized states can make a significant contribution to the PL. The sample (13/10b) is near the type-I to type-II crossover. The PL is dominated by the localized ( $\Gamma 1-\mathrm{hh} 1)$ states below the $\left(X_{z} 1\right.$-hh1) band edge due to their stronger radiative decay. Taken together, the three samples show the increasing contribution of the localized ( $\Gamma 1-\mathrm{hh} 1)$ transitions to the PL with decreasing $\Gamma 1-X_{z} 1$ separation.

The localized type-I and type-II transitions show a different PD due to their different localization sites. We will discuss now how the interface disorder leads to the formation of anisotropically localized type-I transitions with an enhanced PD, while the localized type-II transitions show no significant PD enhancement. The localized $\left(X_{z} 1-\mathrm{hh} 1\right)$ states are sensitive both to the GaAs and AlAs layer thickness fluctuations and are thus not predominantly situated at deeply localized hh1 states, ${ }^{64}$ as is the case for the localized type-I ( $\Gamma 1$-hh1) states. As the hh1 states determine the polarization properties of the transition, the PD of the $\left(X_{z} 1-\mathrm{hh} 1\right)$ transitions is not expected to be affected much by the localization, whereas the PD of the ( $\Gamma 1-\mathrm{hh} 1)$ transitions is enhanced by the anisotropic shape of the deeply localized hh1 states. Additionally, the $\left(X_{z} 1-\mathrm{hh} 1\right)$ transition gains its dipole oscillator strength from the mixing of the $X_{z} 1$ states with the $\Gamma 1$ states due to interface roughness or phonons, predominantly evolving delocalized $\Gamma 1$ states due to their large density of states.

The variation of the PD of the PL over the photon energy in the type-II samples increases when approaching the type-I to type-II transition (Fig. 14), which takes place at about $7 \mathrm{~nm}$ period length [Fig. 8]. This variation is a signature of the enhanced PD of localized ( $\Gamma 1-\mathrm{hh} 1)$ states, visible at photon energies of weak $\left(X_{z} 1\right.$-hh1 $)$ PL. The enhancement is especially pronounced between the zero-phonon energy and the first phonon replica [see, e.g., samples (9/10) and (10/10) in Fig. 13].

The enhanced PD is also present in the PL of the type-I samples [(8/4), (10/5), and (14/5) in Fig. 13], which is dominated by localized ( $\Gamma 1-\mathrm{hh} 1)$ states. Here it shows a maximum approximately at the same photon energy as the PL intensity. The absolute value of the PD maximum is not significantly correlated with the enhancement by a 6-ML APC (dashed lines), and is thus unlikely to originate from a larger corrugation height.

\section{A. Exciton relaxation scheme}

To describe the PL line shape and its dynamics in type-II SL's consistently, we propose the exciton relaxation and re-

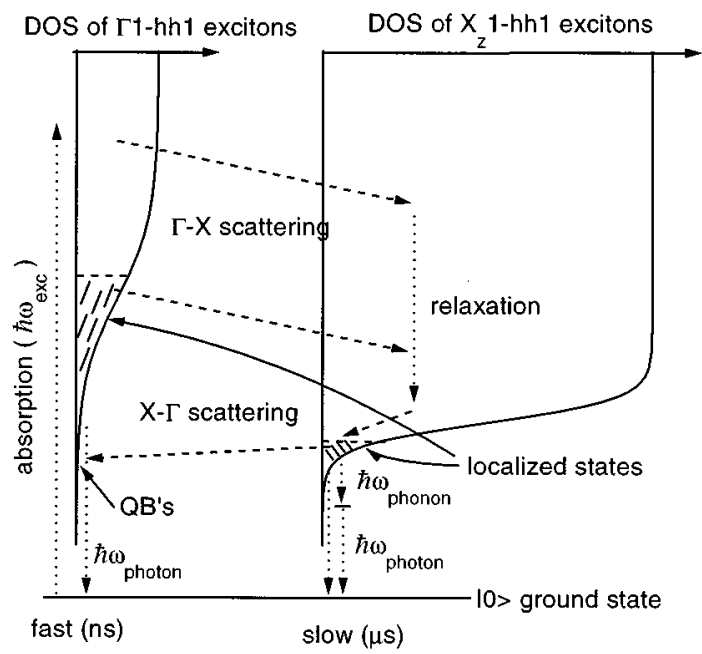

FIG. 16. Relaxation scheme of optically excited electron-hole pairs into the $\Gamma$ quantum boxes, involving the $\left(X_{z} 1-\mathrm{hh} 1\right)$ excitons as transport states.

combination scheme shown in Fig. 16. After the excitation of the electron-hole pairs via the ( $\Gamma 1-\mathrm{hh} 1)$ transition, they relax by the emission of optical and acoustical phonons within some picoseconds to the band edge of the $\left(X_{z} 1-\mathrm{hh} 1\right)$ excitons due to the large density of states of the $X_{z} 1$ subband. The involved scattering processes transfer the electrons from the $\Gamma 1$ to the $X_{z} 1$ subband. The subsequent relaxation dynamics depends strongly on the lattice temperature, the excitation density, and the $\left(\Gamma 1-X_{z} 1\right)$ energy splitting. The $(\Gamma 1-h h 1)$ excitons possess a short radiative recombination time of some $100 \mathrm{ps}$ and a large inhomogeneous width, while the $\left(X_{z} 1\right.$-hh1) excitons feature a long radiative lifetime of some microseconds and about a three times smaller inhomogeneous width. Thus the $\left(X_{z} 1\right.$-hh1) excitons are more mobile than the $(\Gamma 1-\mathrm{hh} 1)$ excitons. This leads, for the $\left(X_{z} 1-\mathrm{hh} 1\right)$ excitons, to a concurrence between their weak radiative recombination and the real-space transport to localized ( $\Gamma 1$-hh1) states, acting as radiative traps. The transport depends strongly on the temperature and the carrier density, while the radiative recombination rate is nearly independent of these quantities. The required transport length is given by the density of localized ( $\Gamma 1-\mathrm{hh} 1)$ states energetically below the $\left(X_{z} 1-\mathrm{hh} 1\right)$ states, which is strongly dependent on the $\left(\Gamma 1-X_{z} 1\right)$ energy splitting. We will demonstrate that the latter states can be addressed as $\Gamma$ quantum boxes (QB's) due to their low density and thus large spatial separation.

\section{B. Samples near the type-II to type-I crossover}

In type-II samples near the type-II to type-I crossover, the low-temperature PL is dominated by the localized ( $\Gamma 1-\mathrm{hh} 1)$ states [see, e.g., samples (13/10a) and (13/10b) in Fig. 13]. Here, the transport to the QB's is possible even at low lattice temperatures due to the high density of QB's, leading to a predominant QB PL with a high-energy cutoff at the $\left(X_{z} 1\right.$-hh1) transition energy. This results in a sharp high energy edge of the PL of the samples. This edge coincides with a dip in the PD and is located at the $\left(X_{z} 1-\mathrm{hh} 1\right)$ transition energy, as determined by the phonon replica positions. The 


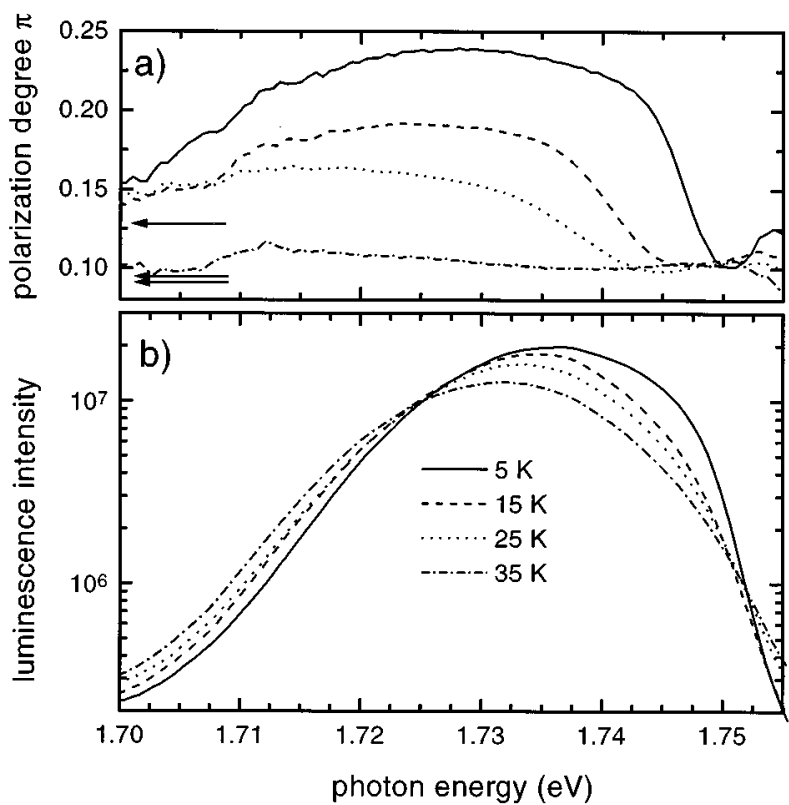

FIG. 17. PD (a) of the PL (b) of the (13/10a) sample for several lattice temperatures, as indicated. The arrows in (a) indicate the calculated values of $0.091,0.095$, and 0.127 for 0,2 , and 6-ML APC, respectively.

phonon replicas also show up in the PD as dips, corresponding to their lower PD compared to the QB's. In the (13/10b) sample, the ( $\Gamma 1$-hh1) transition energy is slightly larger than the $\left(X_{z} 1\right.$-hh1) transition (Fig. 15). In this case, only the exponential tail of the localized ( $\Gamma 1-\mathrm{hh} 1)$ states is situated below the $\left(X_{z} 1-\mathrm{hh} 1\right)$ transition energy, leading to an exponentially decreasing low-energy wing of the PL.

To investigate the influence of higher exciton mobility on the PL, we measured the temperature dependence of the PL and its PD for the (13/10a) sample (Fig. 17). At low temperatures, the $\left(X_{z} 1-\mathrm{hh} 1\right)$ excitons are localized and relax to QB's in their surrounding by phonon-assisted tunneling, as already discussed, giving rise to the sharp high-energy edge of the PL. With increasing temperature, the PL shifts to smaller energies and the high-energy edge broadens. This can be explained by the increasing mobility of the $\left(X_{z} 1\right.$-hh1) excitons due to multiple-trapping processes, allowing for access of a larger spatial area. This results in a trapping rate into the QB's given by the QB density of states and the acoustic phonon-assisted trapping efficiency, which is roughly proportional to the third power of the phonon energy. The result is a redshift and a broadening of the highenergy wing of the PL. Additionally, excitons in QB's with a small energy separation to the $\left(X_{z} 1-\mathrm{hh} 1\right)$ states are detrapped by phonon absorption and their PL is quenched. Simultaneously with the change of the PL line shape, the PD is reduced gradually to the value calculated for the extended states [Fig. 17(a)]. This implies that the QB's, which are excited by the relaxation from localized $\left(X_{z} 1-\mathrm{hh} 1\right)$ states, exhibit an enhanced PD compared to the mean PD of the QB's. The origin of this selectivity is unclear, but might be related to the microscopic arrangement of the localized $\left(X_{z} 1-\mathrm{hh} 1\right)$ states and the QB's. With increasing temperature, only strongly localized $\left(X_{z} 1\right.$-hh1) states remain occupied, leading to the observed redshift of the enhanced PD onset.

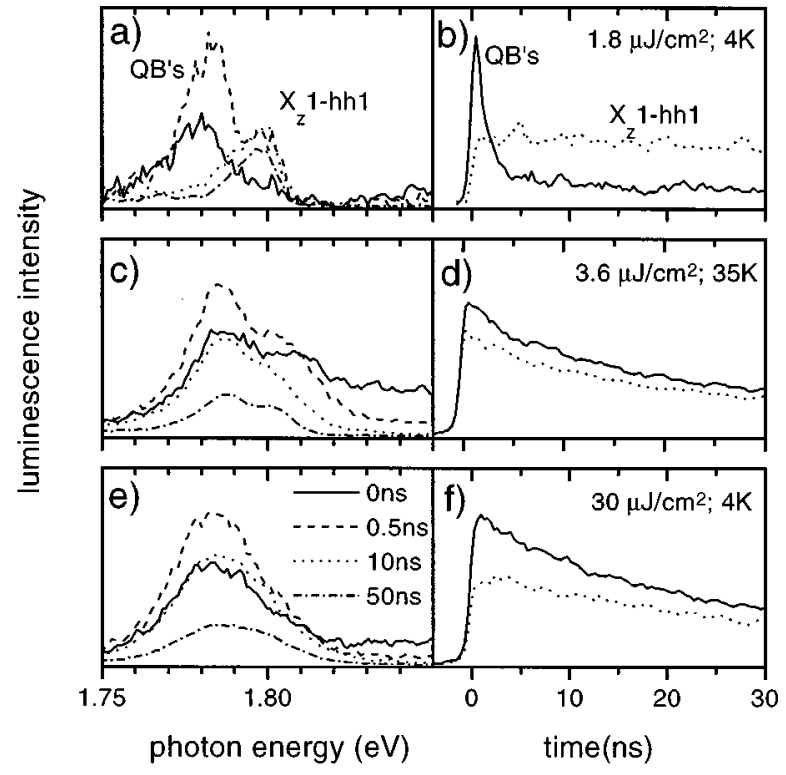

FIG. 18. Time-resolved PL of the (8/8) sample after excitation with 50-ps/2.46-eV pulses. (a), (c), and (e) are spectrally resolved at various times, as given. (b), (d), and (f) are time-resolved intensities of the QB PL (solid lines) and ( $X_{z} 1$-hh1) PL (dotted lines). Excitation intensities and lattice temperatures are given.

\section{Samples with strong type-II character}

In samples with a $\left(\Gamma 1-X_{z} 1\right)$ splitting larger than the ( $\Gamma 1$-hh1) inhomogeneous width [see, e.g., samples (6/4), (7/ 5), (8/8), (9/10), and (10/10) in Fig. 13], the low-temperature PL is dominated by the $\left(X_{z} 1-\mathrm{hh} 1\right)$ transition and its phonon replicas. Here the trapping of the $\left(X_{z} 1-\mathrm{hh} 1\right)$ excitons by the QB's is less efficient due to the small QB density. Only a few of the localized $\left(X_{z} 1-\mathrm{hh} 1\right)$ excitons are situated in the vicinity of a $\mathrm{QB}$. These excitons are trapped into the $\mathrm{QB}$, and recombine subsequently exhibiting the short radiative lifetime of the QB's. The remaining majority of localized $\left(\mathrm{X}_{\mathrm{z}} 1\right.$-hh1) excitons recombine with a long indirect type-II lifetime of a few microseconds. This leads to the dominant $\left(X_{z} 1\right.$-hh1) transition in the PL.

The short-lived QB and long-lived $\left(X_{z} 1-\mathrm{hh} 1\right)$ contribution to the PL can be separated by time resolution, as shown in Fig. 18 for sample (8/8). At low excitation density and lattice temperature [Figs. 18(a) and 18(b)], the $\left(X_{z} 1\right.$-hh1) exciton PL shows a lifetime of more than 100 ns due to its localization and weak radiative decay. Conversely, the lifetime of the QB PL is less than $200 \mathrm{ps}$ due to its type-I character. The evidence of the QB PL in this sample is in agreement with the PD enhancement in the time-integrated PL in between the type-II PL (Fig. 13). At higher temperatures [Figs. 18(c) and 18(d)], the PL of the localized $\left(X_{z} 1-\mathrm{hh} 1\right)$ excitons and of the QB's decay parallel with a decay time of some $10 \mathrm{~ns}$. This is due to the temperature induced mobility within the ( $X_{z} 1$-hh1) states by multiple hopping, leading to a QB PL proportional to the $\left(X_{z} 1-\mathrm{hh} 1\right)$ exciton density. Here the lifetime in the QB's is smaller than the transport time to the QB's, which is again smaller than the decay time of the exciton density. Thus the decay of the QB PL is mainly controlled by the decay of the ( $\left.X_{z} 1-\mathrm{hh} 1\right)$ exciton density via the QB's and nonradiative traps. ${ }^{65}$ 

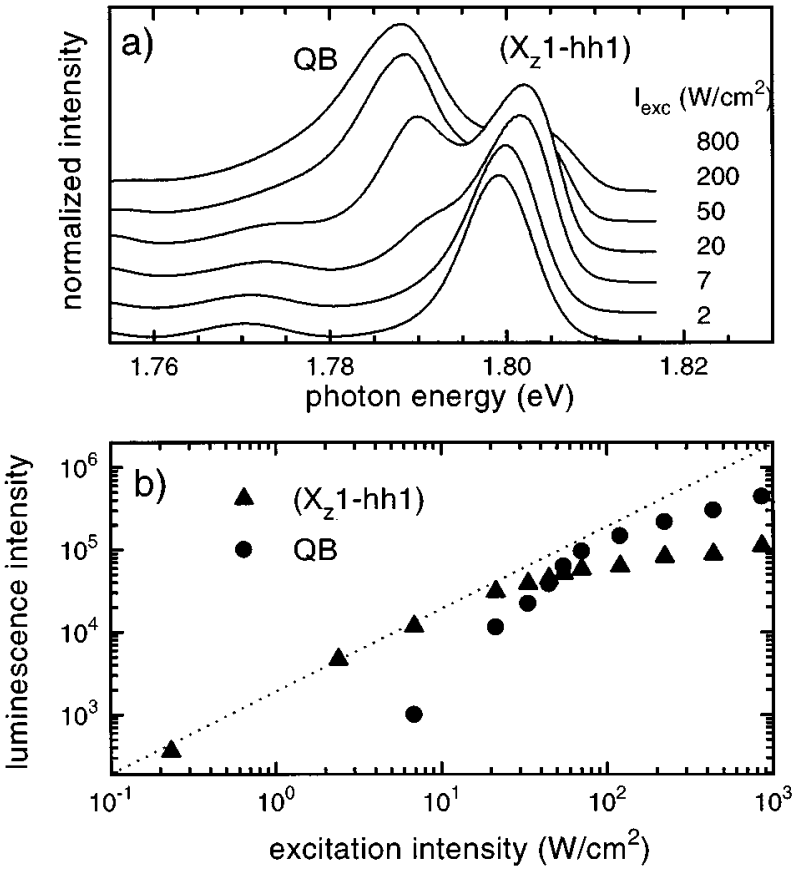

FIG. 19. Time-integrated PL of the (8/8) sample at a excitation at $1.95 \mathrm{eV}$ and a lattice temperature of $2 \mathrm{~K}$. (a) Spectrally resolved, normalized PL for various excitation intensities $I_{\text {exc }}$ as indicated. (b) excitation intensity dependence of the PL intensity $\left(X_{z} 1\right.$-hh1) excitons (triangulars) and QB's (dots). The dotted line indicates a constant PL efficiency.

A similar case evolves at higher excitation densities [Figs. 18 (e) and (f)] of about $1 \times 10^{12} / \mathrm{cm}^{2}$. At this density, the localized $\left(X_{z} 1-\mathrm{hh} 1\right)$ exciton states are occupied, and the mobile delocalized $\left(X_{z} 1\right.$-hh1) excitons are trapped by the QB's. This density dependence of the $\left(X_{z} 1-\mathrm{hh} 1\right)$ exciton mobility is also visible in the time-integrated PL under $\mathrm{cw}$ excitation, shown in Fig. 19(a). With increasing excitation intensity, the $\left(X_{z} 1-\mathrm{hh} 1\right)$ PL shifts to higher energies due to the occupation of localized states. At about $20 \mathrm{~W} / \mathrm{cm}^{2}$, the $\left(X_{z} 1-\mathrm{hh} 1\right) \mathrm{PL}$ intensity and its shift saturates, and the QB PL appears on the low energy wing. The PL efficiency [Fig. 19(b)] starts to decrease at even higher excitation intensities of about $100 \mathrm{~W} / \mathrm{cm}^{2}$, indicating that the mobility is high enough to reach the sparsely distributed nonradiative recombination sites. $^{65}$ This implies that the QB PL is saturated, in agreement with the experimental data [dots in Fig. 19b]. The observed intensity dependence of the PL was previously attributed to the formation of electron-hole droplets. ${ }^{66,67}$ However, the formation dynamics and the temperature dependence of the PL do not support this interpretation.

The influence of the increasing $\left(X_{z} 1\right.$-hh1) exciton mobility with increasing temperatures on the PL and its PD in the type-II sample (9/10) is displayed in Fig. 20. At low temperatures, the PL is dominated by the $\left(\mathrm{X}_{\mathrm{z}} 1-\mathrm{hh} 1\right)$ transition. With increasing temperature, the delocalization of the $\left(\mathrm{X}_{\mathrm{z}} 1-\mathrm{hh} 1\right)$ excitons by phonon absorption leads to an increase of the QB PL intensity. This relaxation channel of the $\left(X_{z} 1\right.$-hh1) excitons becomes dominant over their weak radiative recombination at $15 \mathrm{~K}$ in spite of the small density of the QB's, leading to a prevailing QB PL at $25 \mathrm{~K}$ lattice temperature. This attribution is confirmed by the vanishing intensity

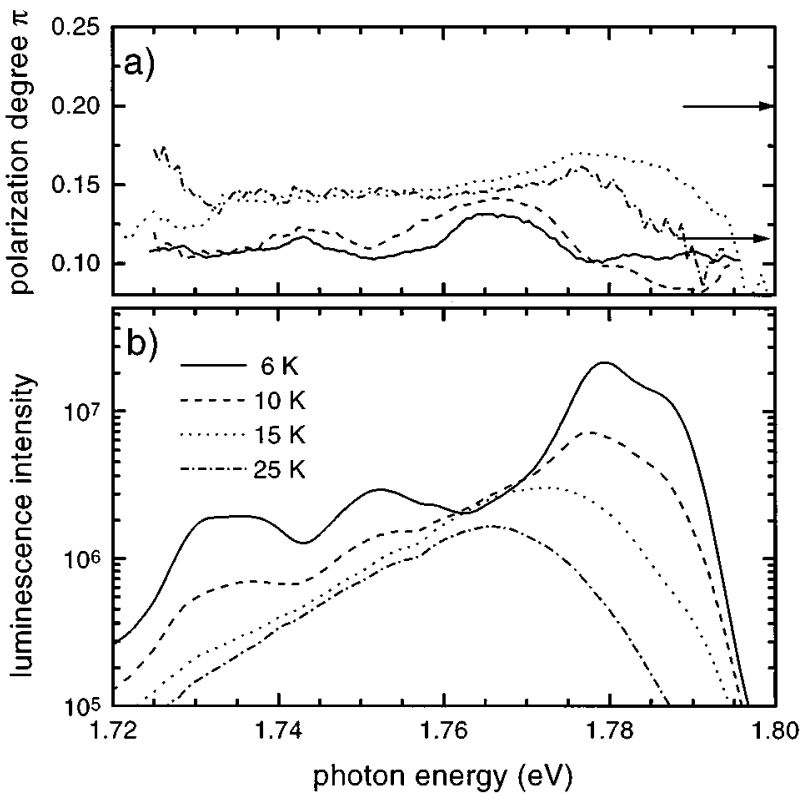

FIG. 20. PD (a) of the PL (b) of sample (9/10) for several lattice temperatures, as indicated. The arrows in (a) indicate the calculated PD's of 0.117 and 0.2 for 0 - and 6-ML APC, respectively.

of the phonon replicas. The simultaneous reduction of the total PL intensity indicates the enhanced trapping of excitons also by nonradiative defects ${ }^{65}$. The PL of the QB's is redshifted in comparison to the $\left(X_{z} 1-\mathrm{hh} 1\right)$ zero-phonon transition, and shows an exponential low energy tail with an slope comparable to the tail in the PLE (compare Fig. 15). This PL shape can be explained by the same underlying mechanisms as previously described for the (13/10) sample. In this case, however, the PD increases with temperature, because the low-temperature PD of the PL is governed here by the PD of the $\left(X_{z} 1\right.$-hh1) states, which is smaller than the PD of the QB's.

\section{Spatially isolated quantum boxes}

To further strengthen our assumption that the QB PL is due to a low density of isolated, deeply localized ( $\Gamma 1-\mathrm{hh} 1)$ states, we have measured the PL of sample (9/10) under high spectral and spatial resolution (Fig. 21). Under these conditions, the emission lines of isolated QB's can be observed individually, showing a spectral width smaller than the instrumental resolution of $0.2 \mathrm{meV}$. The density of the QB's is estimated from the detection radius and the number of lines to be $10^{7} / \mathrm{cm}^{2}$ per SL period. This density is about five orders of magnitude smaller than the band-to-band density of states, in agreement with the value extrapolated from the exponential low energy tail of the PLE spectrum [Fig. 15]. The actual energy positions of the PL lines vary statistically while changing the detection position [Fig. 21(b)]. This confirms that the PL lines originate from isolated localized states with a spatially different localization energy. The temperature dependence of the normalized PL spectra is shown in Fig. 21(c). The QB lines appear with increasing temperature, together with the change of the PL line shape. At temperatures higher than $20 \mathrm{~K}$, the excitons in QB's slightly below the $\left(X_{z} 1\right.$-hh1) states energy become activated, leading to a 


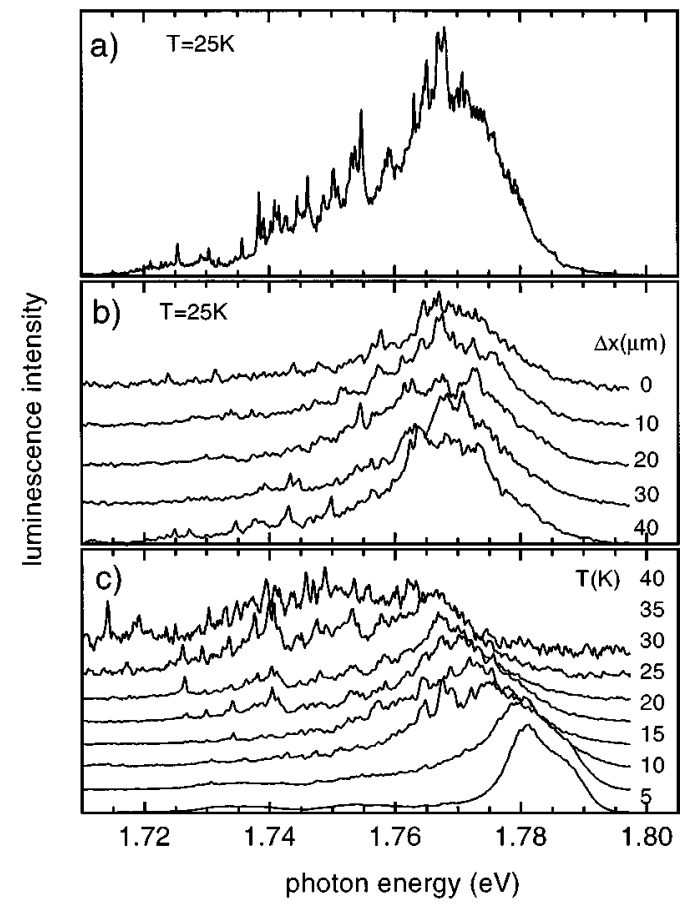

FIG. 21. PL of the (9/10) sample under high spatial resolution at an excitation intensity of $4 \mathrm{~W} / \mathrm{cm}^{2}$ at $1.96 \mathrm{eV}$ using a detection spot diameter of $6 \mu \mathrm{m}$. (a) High spectral resolution at $25 \mathrm{~K}$. (b) Different lateral positions on the sample at $25 \mathrm{~K}$; linear coordinates are given. (c) Normalized PL at different temperatures, as given. The data are vertically offset for clarity.

reduction of the PL intensity (Fig. 20) and a redshift of the PL maximum, as discussed previously.

To obtain information about the shape of the QB's, we analyze their optical anisotropy, which is due to the modified valence-band mixing in the localized hh1 states of an anisotropic geometrical extension. We compare their experimental PL photon energy and PD with the calculated properties of $\mathrm{GaAs}$ quantum wires aligned parallel to the corrugation channels. ${ }^{63}$ This structure model does not exactly reproduce the real shape of the QB's, but gives a reasonable approach to their geometrical properties. By the application of a wire model, the size of the QB's along the [332] direction is as- sumed to be large compared to the size in the other two directions [113] and [110], which is justified by the shape of the interface disorder. ${ }^{25}$

In this model ${ }^{63}$ the experimental transition energy and PD of QB's in samples (13/10a) and (9/10) are met by GaAs quantum wires with a thickness of $3.5 \mathrm{~nm}(4.4 \mathrm{~nm})$ and a width of $10 \mathrm{~nm}$, respectively. This equals a width of three corrugation periods and an additional GaAs thickness of $1.0 \mathrm{~nm}(0.7 \mathrm{~nm})$, equivalent to about 4 (3) times the corrugation height, respectively. This shape is likely to occur in the SL with a 2-ML interface fluctuation. ${ }^{25}$

\section{SUMMARY}

In summary, we have shown that the interface corrugation in (113)-grown GaAs/AlAs SL's does not lead to a quantumwire-like density of states of the lowest conduction and valence subbands, which was claimed to be the reason of several experimental findings such as the anisotropic conductivity, ${ }^{14}$ the enhanced exciton binding energy, ${ }^{13,23}$ and the reduced exciton-exciton scattering. ${ }^{68}$ The comparison of the optical anisotropy of the extended states with the calculation gives an upper limit of $2 \mathrm{ML}$ for the corrugation height present in our samples, in agreement with the surface reconstruction proposed by Wassermeier et al. ${ }^{25}$ and Raman experiments. ${ }^{28}$ The strongly anisotropic properties in the conductivity and in the optical properties of the PL in these SL's are most likely due to the anisotropic interface disorder, which is developing in the presence of the interface corrugation. This gives rise to anisotropically localized electronic states at the band edges. We have demonstrated the existence of deeply localized, anisotropic quantum boxes in these SL's, which show very narrow emission lines with an enhanced optical anisotropy. The shape of these quantum boxes of the type-I transition is estimated using a quantum-wire model to be about three corrugation periods in width and 4 ML in additional height.

\section{ACKNOWLEDGMENTS}

The authors want to thank C. Klingshirn and D. Birkedal for helpful discussions and A. Fischer for technical assistance in MBE growth. This work was supported by the Deutsche Forschungsgemeinschaft and by the Danish Ministries of Research and Industry in the framework of CNAST.
*Present address: Mikroelektronik Centret, DTU, Bldg. 345 east, DK-2800 Lyngby, Denmark. E-mail: Langbein@mic.dtu.dk

${ }^{1}$ J.S. Weiner et al., Phys. Rev. Lett. 63, 1641 (1989).

${ }^{2}$ E. Kapon, D.M. Wang, and R. Bhat, Phys. Rev. Lett. 63, 430 (1989).

${ }^{3}$ M.A. Reed et al., Phys. Rev. Lett. 60, 535 (1988).

${ }^{4}$ M. Kohl, D. Heitmann, P. Grambow, and K. Ploog, Phys. Rev. Lett. 63, 2124 (1989).

${ }^{5}$ J. Cibert et al., Appl. Phys. Lett. 49, 1275 (1986).

${ }^{6}$ M. Notomi, M. Okamoto, H. Iwamura, and T. Tamamura, Appl. Phys. Lett. 62, 1094 (1993).

${ }^{7}$ F.E. Prins et al., Appl. Phys. Lett. 62, 1365 (1993).

${ }^{8}$ D. Gershoni et al., Phys. Rev. Lett. 65, 1631 (1990).

${ }^{9}$ H.L. Störmer et al., Appl. Phys. Lett. 58, 726 (1991).

${ }^{10}$ A.R. Goñi et al., Appl. Phys. Lett. 61, 1956 (1992).
${ }^{11}$ T. Someya, H. Akiyama, and H. Sakaki, Appl. Phys. Lett. 66, 3672 (1995).

${ }^{12}$ T. Sogawa, S. Ando, H. Ando, and H. Kanbe, Appl. Phys. Lett. 68, 364 (1996).

${ }^{13}$ R. Nötzel et al., Phys. Rev. Lett. 67, 3812 (1991).

${ }^{14}$ R. Nötzel et al., Phys. Rev. B 45, 3507 (1992).

${ }^{15}$ R. Nötzel, L. Däweritz, and K. Ploog, Phys. Rev. B 46, 4736 (1992).

${ }^{16}$ E. Tournié, R. Nötzel, and K. Ploog, Phys. Rev. B 49, 11053 (1994).

${ }^{17}$ V.A. Shchukin, A.I. Borovkov, N.N. Ledentsov, and P.S. Kop'ev, Phys. Rev. B 51, 17767 (1995).

${ }^{18}$ F. Briones, L. Gonzáles, and A. Ruiz, Appl. Phys. A 49, 729 (1989).

${ }^{19}$ Y. Hsu, W.I. Wang, and T.S. Kuan, Phys. Rev. B 50, 4973 (1994). 
${ }^{20}$ G. Armelles et al., Phys. Rev. B 49, 14020 (1994).

${ }^{21}$ A.J. Shields et al., Appl. Phys. Lett. 60, 2537 (1992).

${ }^{22}$ Z.V. Popović et al., Phys. Rev. B 52, 5789 (1995).

${ }^{23}$ R. Nötzel, N.N. Ledentsov, and K. Ploog, Phys. Rev. B 47, 1299 (1993)

${ }^{24}$ Z.Y. Xu et al., Phys. Rev. B 51, 7024 (1995).

${ }^{25}$ M. Wassermeier et al., Phys. Rev. B 51, 14721 (1995).

${ }^{26}$ M. Wassermeier et al., J. Cryst. Growth 150, 425 (1995).

${ }^{27}$ W. Braun et al., Appl. Surf. Sci. (to be published).

${ }^{28}$ S. da Silva et al., Phys. Rev. B 53, 1927 (1996).

${ }^{29}$ W. Langbein et al., Nuovo Cimento 17D, 1567 (1995).

${ }^{30}$ M.V. Belousov et al., Phys. Solid State 36, 596 (1994).

${ }^{31}$ P.V. Santos et al., Phys. Rev. B 52, 1970 (1995).

${ }^{32}$ E. Ribeiro, F. Cerdeira, and A. Cantarero, Phys. Rev. B 51, 7890 (1995).

${ }^{33}$ J.-B. Xia, Phys. Rev. B 43, 9856 (1991).

${ }^{34}$ D. Gershoni et al., Phys. Rev. B 44, 1930 (1991).

${ }^{35}$ S. Nojima, Phys. Rev. B 47, 13535 (1993).

${ }^{36}$ E.C. Valadares, Phys. Rev. B 46, 3935 (1992).

${ }^{37}$ D.A. Contreras-Solorio, V.R. Velasco, and F. Garćia-Moliner, Phys. Rev. B 47, 4651 (1993).

${ }^{38}$ C. Jouanin, A. Hallaoui, and D. Bertho, Phys. Rev. B 50, 1645 (1994).

${ }^{39}$ Z. Ikonić, G.P. Srivastava, and J.C. Inkson, Phys. Rev. B 49, 10749 (1994).

${ }^{40}$ R.K. Hayden et al., Phys. Rev. B 46, 15586 (1992).

${ }^{41}$ R.K. Hayden et al., Semicond. Sci. Technol. 9, 298 (1994).

${ }^{42}$ G. Armelles et al., J. Phys. IV 3, 283 (1993).

${ }^{43}$ C. Jouanin and D. Bertho, J. Phys. IV 3, 287 (1993).

${ }^{44}$ A.A. Kiselev and U. Rössler, Phys. Rev. B 50, 14283 (1994).

${ }^{45}$ W. Langbein, Ph.D. thesis, Universität Karlsruhe, Karlsruhe, 1995.
${ }^{46}$ S.-S. Li and J.-B. Xia, Phys. Rev. B 50, 8602 (1994).

${ }^{47}$ J.-B. Xia and S.-S. Li, Phys. Rev. B 51, 17203 (1995).

${ }^{48}$ B. Devaud et al., Phys. Rev. B 40, 5802 (1989).

${ }^{49}$ K. Fujiwara et al., Phys. Rev. B 49, 1809 (1994).

${ }^{50}$ G. Oelgart et al., Phys. Rev. B 49, 10456 (1994).

${ }^{51}$ E.S. Koteles and J.Y. Chi, Phys. Rev. B 37, 6332 (1994).

${ }^{52}$ V. Voliotis, R. Grousson, P. Lavallard, and R. Planel, Phys. Rev. B 52, 10725 (1995).

${ }^{53}$ J.M. Luttinger, Phys. Rev. 102, 1030 (1956).

${ }^{54}$ G. Edwards, E. Valdares, and F.W. Sheard, Phys. Rev. B 50, 8493 (1994).

${ }^{55}$ S. Nojima, Jpn. J. Appl. Phys. 31, L1401 (1992).

${ }^{56}$ P. Lawaetz, Phys. Rev. B 4, 3460 (1971).

${ }^{57}$ E.G. Tsitsishvili, Phys. Rev. B 52, 11172 (1995).

${ }^{58}$ Y. Kajikawa, Phys. Rev. B 51, 16790 (1995).

${ }^{59}$ R. Winkler and A.I. Nesvizhskii, Phys. Rev. B 53, 9984 (1996).

${ }^{60}$ P. Lefebvre, B. Gil, H. Mathieu, and R. Planel, Phys. Rev. B 39, 5550 (1989).

${ }^{61}$ H. van Kesteren et al., Phys. Rev. B 39, 13426 (1989).

${ }^{62}$ L.P. Fu et al., Phys. Rev. B 52, 2682 (1995).

${ }^{63}$ W. Langbein et al., Phys. Rev. B 53, 15473 (1996).

${ }^{64}$ A.B. Dzyubenko and G. E. W. Bauer, Phys. Rev. B 51, 14524 (1995).

${ }^{65}$ G.D. Gilliland et al., Phys. Rev. Lett. 71, 3717 (1993).

${ }^{66}$ H. Kalt, R. Nötzel, K. Ploog, and H. Giessen, Solid State Commun. 83, 285 (1992).

${ }^{67}$ H. Kalt, J. Lumin. 60, 262 (1994).

${ }^{68}$ D.S. Jiang et al., Phys. Rev. B 49, 10786 (1994).

${ }^{69}$ Properties of Aluminium Gallium Arsenide, edited by S. Adachi (INSPEC, London, 1988). 